\title{
Impact of soil and foliar fertilization on yield and net income of maize plants alluvial clay soil.
}

\author{
**Ahmed M. M. Hassanien * Esmat H. A. Noufal * Omar H. M. El-Husseini ** Safwat A. Ismail \\ * Fac. of Agric. Benda Univ. - ** Agricultural Research Center (A.R.C.)
}

Corresponding author: esmat.noufal@yahoo.com

\begin{abstract}
The study was aimed to investigate the effect of methods and levels of nitrogen, phosphorus and potassium application on maize growth, yield components, yields, nutrient uptake as well as net income. Two field experiments were carried out in the at Agricultural Farm of Sids Agricultural Research Station, ARC, Beni - Swif Governorate, Egypt during the two successive seasons of 2013 and 2014.

The nitrogen treatments were $120 \mathrm{~kg} \mathrm{~N} / \mathrm{fed}$ as soil application $\left(\mathrm{N}_{1}\right), 90 \mathrm{~kg} \mathrm{~N} / \mathrm{fed}$ as soil application + two time foliar spraying of $2 \%$ urea solution $\left(\mathrm{N}_{2}\right)$ and $60 \mathrm{~kg} \mathrm{~N} / \mathrm{fed}$ as soil application + two time foliar spraying of $2 \%$ urea solution $\left(\mathrm{N}_{3}\right)$. The phosphorus treatments were $13.6 \mathrm{~kg} \mathrm{P} / \mathrm{fed}$ as soil application $\left(\mathrm{P}_{1}\right), 6.8 \mathrm{~kg} \mathrm{P} / \mathrm{fed}$ as soil application + two time foliar spraying of $0.33 \%\left(\mathrm{P}_{2}\right)$, and two time foliar spraying of $0.33 \% \mathrm{P}\left(\mathrm{P}_{3}\right)$. The potassium treatments were $39.8 \mathrm{~kg} \mathrm{~K} /$ fed as soil application $\left(\mathrm{K}_{1}\right), 19.9 \mathrm{~kg} \mathrm{~K} / \mathrm{fed}$ as soil application + two time foliar spraying of $2 \%$ potassium sulphate solution $\left(\mathrm{K}_{2}\right)$, and two time foliar spraying of $2 \%$ potassium sulphate solution $\left(\mathrm{K}_{3}\right)$. A factorial experiment design included 27 treatments with four replications was performed. The results reveal that maize plant height, dry weight/plant, number of rows / ear , number of grains/ row , 100 - grain weight, grain , stover and biological yields as well as nutrient uptake and net income were significantly responded to methods and levels of N,P and K application. On the other hand combined soil application with foliar spraying (90 kg N/ fed with foliar spraying of $2 \%$ urea solution, $6.8 \mathrm{~kg} \mathrm{P} / \mathrm{fed}$ with foliar spraying of $0.33 \% \mathrm{P}$ or $19.9 \mathrm{~kg} \mathrm{~K} / \mathrm{fed} \mathrm{with}$ foliar spraying of $2 \%$ potassium sulphate solution) gave higher values, of growth parameter except plant height with $120 \mathrm{~kg} \mathrm{~N} / \mathrm{fed}$ which recorded the tallest maize plant. In general, the recommended treatment for quality and quantity as well as the net income of maize was $90 \mathrm{~kg} \mathrm{~N} / \mathrm{fed}$ as soil application in combination with foliar spraying of $2 \%$ urea solution $+6.8 \mathrm{~kg}$ P/fed as soil application in combination with foliar spraying of $0.33 \% \mathrm{P}+19.9 \mathrm{~kg}$ $\mathrm{K} /$ fed as soil application in combination with foliar spraying of $2 \%$ potassium sulphate solution.
\end{abstract}

Key words: NPK fertilization, soil application, foliar spraying, growth parameters, yield and its components, nutrient uptake and net income.

\section{Introduction}

Maize (Zea mays L.) is the most important cereal crops grown in the world after wheat and rice with respect to the area and principally. It is an important source of carbohydrate, protein, iron, vitamin B and minerals. It is the most important cereal fodder and grain crop under both irrigated and rainfed agricultural systems in the semi - arid and arid tropics. Maize has numerous uses and ranks second only to wheat among the worlds cereal crops in terms of total production. Also, because of its worldwide distribution and lower price, relative to other cereals, maize has a wide range of uses than any other cereals. It is the stable food crop and the base of most rural diets, as well as a cash crop. In poor communities, it is the main source of calories and protein, as well as the primary weaning food for babies. In developed countries, maize is consumed mainly as second.

The new high yielding hybrids will be adapted to different cultural practices such. Also, in Egypt, intensive cropping has become more common and $\mathrm{N}$, $\mathrm{P}$ and $\mathrm{K}$ fertilizers the primary function of soil productivity and fertility restoration has become less effective. On the other hand, most of soil in Egypt are known to be poor in fertility status, hence it is need to supplement the amount of nutrients for optimal crop performance. The poor fertility status is even more related to chemical than physical properties (Fernandez and Sanche, 1990). Though the nutrients such as N, P and K supplied by mineral fertilizers are usually specific and released rather fast.

Nitrogen is a vital plant nutrient and major determining factor required for maize production. It is very essential for plant growth and makes up 1-4 \% of the dry matter of plants. Nitrogen is a component of protein and nucleic acids and also an integral component of many other compounds essential for plant growth processes including chbrophyll and many enzymes. Gebrail et al (2005), Siam et al (2008), Ali et al (2012) and Thirupathi, et al (2016) reported that added nitrogen as soil application improved growth and yield and its components on maize plant .Ismail et al (2006), Sarhan (2006), Sadik et al (2009) and Abd El-Hafeez et al (2013) found that $\mathrm{N}, \mathrm{P}$ and $\mathrm{K}$ uptake in maize grains and stover were significantly responded to soil nitrogen application .

Phosphorus is another essential nutrient required to increase maize productivity. Phosphorus plays an important role in many physiological processes, involved in enzymatic reactions in plant, essential factor for cell divition because it is constituent element of nucleoproteins which are involved in the cell reproduction processes, a component of a chemical essential to the reactions of carbohydrate synthesis and degradation, important for seed and 
fruit formation and crop maturation as well as affects quality of the grains and it may increase the plant resistance to diseaces. Hussain et al (2007), Yosefi et al (2011) and Amahakiam and Osemwota (2012) stated that supplied maize plant with phosphorus as soil application resulted in a positive effect on growth and yield and yield components. Kruczek (2005) and Crozier et al (2009) indicated that phosphorus added to soil enhanced phosphorus concentration and uptake in maize.

Potassium is, one of the essential nutrient in plant. It plays essential roles in enzyme activation, protein synthesis, photosynthesis, osmoregulation, stomatal movement, energy transfer, cation - anion balance and stress resistance. Maize productivity were increased due to potassium soil application (Sedrak, 2007 and Maqsood et al 2013). Also, nutrients status of maize were increased due to potassium soil application (Kubar et al .2013 and Zorkany, 2014).

Foliar feeding has been used as a mean of supplying supplemental doses of macro and micro nutrients, plant hormones stimulants and beneficial substances. Observed effects of foliar fertilization have included yield increases, resistance to plant diseaces and insect, improved drought tolerance and enhanced crops quality. In term of nutrient absorption, foliar fertilization can absorbed from 8 to 20 times as efficient as ground application (Anonymous 1985). Parasuraman et al (2008), Hu et al (2008), Khan et al (20016) reported that maize productivity improved due to foliar spraying of N P K fertilizers.

This study was conducted to determine the yield and growth of maize plant as affected by N, P and K fertilization as soil application as well as supplement part of them as foliar spraying.

\section{Materials and Methods}

The current study was conducted at the Agricultural Farm of Sids Agricultural Research Station, ARC, Beni-Swif Governorate, Egypt during the two summer seasons of 2013 and 2014 to evaluate the effect of applied NPK fertilizers as soil or foliar application on plant growth parameters, yield and yield compents, NPK uptake and the net income of maize plant .The experimental design was a randomized com plate block factorial involving three factors as follows : a , b , c, Each treatment was replicated four times .

\section{The factors were.}

Factor A Nitrogen fertilizer. $120 \mathrm{~kg} \mathrm{~N} /$ fed as soil application $\left(\mathrm{N}_{1}\right), 90 \mathrm{~kg} \mathrm{~N} / \mathrm{fed}$ as soil application + foliar spraying of $2 \%$ urea solution twice $\left(\mathrm{N}_{2}\right)$ and 60 $\mathrm{kg} \mathrm{N} /$ fed as soil application + foliar spraying of $2 \%$ urea solution twice $\left(\mathrm{N}_{3}\right)$.

Factor B Phosphorus fertilizer. $13.6 \mathrm{~kg} \mathrm{P} / \mathrm{fed}$ as soil application $\left(\mathrm{P}_{1}\right)$ in form of superphosphate $(15.5$ $\left.\mathrm{P}_{2} \mathrm{O}_{5}\right), 6.8 \mathrm{~kg} \mathrm{P} / \mathrm{fed}$ as soil application + foliar spraying of $0.33 \% \mathrm{P}$ as the supernatant of 10 and 20 $\mathrm{kg} /$ fed superphosphate soaking for the first and second spraying, respectively twice $\left(\mathrm{P}_{2}\right)$ and foliar spraying of $0.33 \% \mathrm{P}$ as the supernatant of 10 and $20 \mathrm{~kg} / \mathrm{fed}$ superphosphate soaking for the first and second spraying, respectively twice $\left(\mathrm{P}_{3}\right)$.

Factor C Potassium fertilizer. $39.8 \mathrm{~kg} \mathrm{~K} / \mathrm{fed}$ as soil application $\left(\mathrm{K}_{1}\right)$ in form of potassium sulphate $48 \%$ $\mathrm{K}_{2} \mathrm{O}, 19.9 \mathrm{~kg} \mathrm{~K} / \mathrm{fed}$ as soil application + foliar spraying of $2 \%$ potassium sulphate solution twice $\left(\mathrm{K}_{2}\right)$ and foliar spraying of $2 \%$ potassium sulphate solution twice $\left(\mathrm{K}_{3}\right)$.

The preceding crop was wheat in the two studied seasons. The plot area was $3 \times 3.5 \mathrm{~m}=1 / 400 \mathrm{fed}$. Each experimental plot consisted of five rows, three meters along and $0.70 \mathrm{~m}$ apart. Grains of maize (Zea mays L.), Single Cross 10 were sowing in hills, $0.30 \mathrm{~m}$ apart in 11 and 17 May in the two seasons, respectively. Thinning was done before the first irrigation to one plant/hill which gave about 20000 plant/fed. Other cultural practices for maize production were done as in district.

The total number treatment war $3 \times 3 \times 3 \times 4=108$ plot.

The soil application of nitrogen fertilizer was added at two equal doses, as ammonium nitrate , $33.5 \% \mathrm{~N}$, the first before the first irrigation and the second before the second irrigation while nitrogen foliar spraying was done as urea $46.5 \% \mathrm{~N}$. On the other hand, soil application of phosphorus fertilizer was done before planting during the land preparation, anal potassium soil application was done at two equal doses, the first before planting during the land preparation and the other after one month from maize sowing. However, the foliar spraying of N, P or K war done performed tow time, the first after the first irrigation and the second one after one month later at a rate of 200 and $400 \mathrm{~L} / \mathrm{fed}$ in the two foliar application, respectively.

\section{Data recorded:}

Five plants were randomly taken from each plot during tasseling - silking stage (about 60 days age) from the two inner rows to measure some growth characters such as:

1- Plant height $(\mathrm{cm})$

2- Dry weight / plant (g)

3- Number of leaves /plant

4- Leaf area $\left(\mathrm{cm}^{2}\right)$

At harvesting, five plants were randomly taken from each plot from the two inner rows to measure some yield components such as:

1-The number of rows/ ear

2-The number of grains / row

3-100 - grain weight

Also, grain and stover yields were measured for each plot and calculated as $\mathrm{Mg} / \mathrm{h}^{-1}$

Nitrogen, phosphorus and potassium percent in both grains and stover were determined according to the method described by Chapman and Pratt (1961) and nutrient uptake was calculated. 
Net income was calculated as:

Net income $=$ price of weight of maize grains price of added fertilizer

The average price of maize grains /t was about 3000 L.E in the two season

The average price of one $\mathrm{kg} \mathrm{N}$ from urea and ammonium nitrate were 6.37 and 8.84 L.E, respectively (2960 L.E/t for each)

The average price of one $\mathrm{kg} \mathrm{P}$ was $19.8 \mathrm{L.E}$ (1340 L.E/t) from superphosphate fertilizer $(0.33 \% \mathrm{P})$
The average price of one $\mathrm{kg} \mathrm{K}$ was $19.33 \mathrm{L.E}$ (7700 L.E/t) from potassium sulphate fertilizer $(48 \%$ $\mathrm{K}_{2} \mathrm{O}$ )

The cost of fertilizer application workers was neglected because the cost of soil application is somewhat equal to those for foliar spraying.

\section{Soil analysis:}

Representive soil sample (0-30) was taken before planting from the experiment sites in the two seasons to determine some physical ( according to Piper, 1950 ) and chemical ( according to the standard methods cited by Jacson, 1967) Soil properties and listed in Table (1) as follows:

Table 1. Physical and chemical properties of the experimental soil.

\begin{tabular}{|c|c|c|}
\hline Property & First season & Second season \\
\hline \multicolumn{3}{|l|}{ Particle size distribution } \\
\hline Sand $\%$ & 17.22 & 15.71 \\
\hline Silt $\%$ & 29.13 & 31.08 \\
\hline Clay $\%$ & 53.65 & 53.21 \\
\hline Texture class & clay & clay \\
\hline $\mathrm{pH}$ ( in soil paste extract) & 8.0 & 8.1 \\
\hline EC dSm -1 ( in soil paste extract ) & 1.31 & 1.35 \\
\hline Organic matter $\mathrm{g} \mathrm{kg}^{-1}$ & 13.5 & 12.0 \\
\hline \multicolumn{3}{|l|}{ Soulble cations $\mathrm{m}$ mole $\mathrm{L}^{-1}$} \\
\hline $\mathrm{Ca}^{2+}$ & 4.71 & 4.85 \\
\hline $\mathrm{Mg}^{2+}$ & 4.38 & 4.49 \\
\hline $\mathrm{Na}^{+}$ & 2.11 & 2.29 \\
\hline $\mathrm{K}^{+}$ & 1.80 & 1.85 \\
\hline \multicolumn{3}{|l|}{ Soulble anions $\mathrm{m}$ mole $\mathrm{L}^{-1}$} \\
\hline $\mathrm{CO}_{2}^{-}$ & -- & -- \\
\hline $\mathrm{HCO}_{3}^{-}$ & 1.61 & 1.75 \\
\hline CI- & 4.92 & 4.96 \\
\hline $\mathrm{SO}_{4}^{-}$ & 6.47 & 6.77 \\
\hline Available $\mathrm{N} \mathrm{mg} \mathrm{kg}{ }^{-1}$ & 25.3 & 27.1 \\
\hline Available $\mathrm{P} \mathrm{mg} \mathrm{kg}^{-1}$ & 10.7 & 9.5 \\
\hline Available $\mathrm{K} \mathrm{mg} \mathrm{kg}^{-1}$ & 170 & 182 \\
\hline
\end{tabular}

\section{Results and Discussion}

\section{Growth parameters.}

Data presented in Tables 2, 3, 4 and 5 show the effect of applied nitrogen, phosphorus and potassium fertilization on maize growth at 60 days from planting namely, plant height, dry weight/plant, number of leaves / plant and leaf area. The results show that nitrogen treatments $\left(\mathrm{N}_{1}, \mathrm{~N}_{2}\right.$ and $\left.\mathrm{N}_{3}\right)$ had significant effects on maize growth, except the number of leaves / plant. The highest values of the studied parameters were obtained under $120 \mathrm{~kg} \mathrm{~N} /$ fed as soil application $\left(\mathrm{N}_{1}\right)$, whereas added $60 \mathrm{~kg} \mathrm{~N} / \mathrm{fed}$ as soil application plus foliar spraying of $2 \%$ urea solution twice $\left(\mathrm{N}_{3}\right)$ exhibited the lowest ones. Furthermore, $\left(\mathrm{N}_{1}\right)$ statistically gave dry weight / plant equal to that obtained under $90 \mathrm{~kg} \mathrm{~N} / \mathrm{fed}$ as soil application + tow time foliar spraying of $2 \%$ urea solution $\left(\mathrm{N}_{2}\right)$. The increase in maize growth due to increasing the $\mathrm{N}$ level up to $120 \mathrm{~kg} /$ fed is mainly due to the vital role of nitrogen in plant growth, as it is necessary for protoplasm formation and photosynthesis in all plant, it is also necessary for cell devition and merestimatic activity in organs. These results are similar to those obtained by Sarhan (2006) and Sadik et al (2009) who stated that maize plants was increased as nitrogen fertilization increased.

As for phosphorus or potassium treatments the obtained results reveal that plant height, dry weight/plant and leaf area were positively responded to the added $\mathrm{P}_{1}$ (13.6 $\mathrm{kg} \mathrm{P} /$ fed as soil application) or $\mathrm{P}_{2}(6.8 \mathrm{~kg} \mathrm{P} / \mathrm{fed}$ as soil application + tow time foliar spraying of $0.33 \% \mathrm{P})$ as well as $\mathrm{K}_{1}(39.9 \mathrm{~kg} \mathrm{~K} /$ fed as soil application) or (19.9 kg K/fed as soil application + tow time foliar spraying of $2 \%$ potassium sulphate solution ). Whereas the, number of leaves / plant did not respond to phosphorus or potassium treatments. The beneficial of mixing soil application with foliar spraying is mainly due to the fact that foliar spraying method provide rapid nutrient utilization and enables quick correction of nutrient difficiencies, However, the response fo foliar fertilization is often temporary 
due to minute amount of applied nutrient (Halvin et al, 2005). The obtained results are in agreement with those obtained by . While, the positive effect of increasing potassium levels $\left(\mathrm{K}_{1}\right)$ is mainly due to potassium is an essential macronutrient, it plays essential roles in enzyme activation, protein photosynthesis, osmo regulation, stomata movement, energy transfer, phloem transport, cation - anion balance, and stress resistance (Mingle and kiry, 1987) . These results agree with those obtained by Sidrak (2007) who stated that growth parameter of maize was significantly affected by increasing potassium rates.

On the other hand, $\mathrm{N}_{3}(60 \mathrm{~kg} / \mathrm{fed}$ as soil application + foliar spraying of $2 \%$ urea solution twice), $\mathrm{P}_{3}$ (foliar spraying of $0.33 \% \mathrm{P}$ twice) or $\mathrm{K}_{3}$ ( tow time foliar spraying of $2 \%$ potassium solution ) treatment yield gave the lowest values of plant height, dry weight / plant and leaf area of maize plant as compared with $\mathrm{N}_{1}, \mathrm{~N}_{2}+\mathrm{sp}, \mathrm{P}_{1}, \mathrm{P}_{2}+{ }^{\mathrm{S} P}$ and $\mathrm{K}_{1}, \mathrm{~K}_{2}+{ }^{\mathrm{S} P}$, respectively

The interaction between treatments indicate that the treatment of $\mathrm{N}_{1}+\mathrm{P}_{1} \mathrm{O}+\mathrm{K}_{1}$ gave the highest plant height, leaf area and dry weight of maize plant the other hand, the treatment of $\mathrm{N}_{3}+\mathrm{P}_{3}+\mathrm{K}_{3}$ gave the lowest values of the abovementioned growth parameters.

Table 2 . Effect of soil and foliar application of different N,P and K levels on plant height of 60 days from planting $(\mathrm{cm})$

\begin{tabular}{|c|c|c|c|c|c|c|c|c|c|}
\hline \multirow{3}{*}{ Nitrogen } & \multirow{3}{*}{ Phosphorus } & \multicolumn{8}{|c|}{ Potassium } \\
\hline & & \multicolumn{4}{|c|}{ First season } & \multicolumn{4}{|c|}{ second season } \\
\hline & & $\mathbf{K}_{1}$ & $\mathbf{K}_{2}$ & $\mathbf{K}_{3}$ & Mean & $\mathbf{K}_{1}$ & $\mathbf{K}_{2}$ & $\mathbf{K}_{3}$ & Mean \\
\hline \multirow{3}{*}{$\mathbf{N}_{1}$} & $\mathbf{P}_{1}$ & 185.22 & 184.04 & 176.30 & 181.85 & 183.19 & 181.35 & 178.50 & 181.01 \\
\hline & $\mathbf{P}_{2}$ & 183.40 & 183.10 & 175.80 & 180.77 & 180.94 & 180.09 & 177.30 & 179.44 \\
\hline & $\mathbf{P}_{3}$ & 177.50 & 176.80 & 173.20 & 175.83 & 177.90 & $\mathbf{1 7 7 . 3 0}$ & 173.50 & 176.23 \\
\hline \multicolumn{2}{|l|}{ Mean } & 182.04 & 181.31 & 175.10 & 179.15 & 180.68 & 179.58 & 176.43 & 178.89 \\
\hline \multirow{3}{*}{$\mathbf{N}_{2}$} & $\mathbf{P}_{1}$ & 180.11 & 179.33 & 175.60 & 178.35 & 179.00 & 178.60 & 175.5 & 177.7 \\
\hline & $\mathbf{P}_{2}$ & 179.22 & 178.60 & 175.30 & 177.71 & 178.30 & 177.90 & 175.00 & 177.07 \\
\hline & $\mathbf{P}_{3}$ & 175.20 & 174.80 & 171.40 & 173.80 & 174.82 & 175.30 & 172.91 & 174.34 \\
\hline \multicolumn{2}{|l|}{ Mean } & 178.18 & 177.58 & 174.10 & 176.62 & 177.37 & 177.27 & 174.47 & 176.37 \\
\hline \multirow{3}{*}{$\mathbf{N}_{3}$} & $\mathbf{P}_{1}$ & 174.11 & 173.90 & 167.30 & 171.77 & 173.55 & 172.10 & 168.46 & 171.37 \\
\hline & $\mathbf{P}_{2}$ & 173.80 & 173.30 & 166.50 & 171.20 & 171.41 & 171.09 & 167.79 & 170.10 \\
\hline & $\mathbf{P}_{3}$ & 168.30 & 167.5 & 163.22 & 166.34 & 168.46 & 167.79 & 165.11 & 167.12 \\
\hline \multicolumn{2}{|l|}{ Mean } & 172.07 & 171.57 & 165.67 & 169.77 & 171.14 & 170.33 & 167.12 & 169.53 \\
\hline \multirow{3}{*}{ Mean of $P$} & $\mathbf{P}_{1}$ & 179.81 & 179.09 & 173.07 & 177.32 & 178.58 & 177.35 & 174.15 & 176.69 \\
\hline & $\mathbf{P}_{2}$ & 178.81 & 178.33 & 172.53 & 176.56 & 176.88 & 176.36 & 173.36 & 175.53 \\
\hline & $\mathbf{P}_{3}$ & 173.67 & 173.03 & 169.27 & 171.99 & 173.73 & 173.96 & 170.51 & 172.56 \\
\hline \multicolumn{2}{|l|}{ Mean of $K$} & 177.43 & 176.82 & 171.62 & 175.29 & 176.40 & 175.72 & 172.63 & 174.93 \\
\hline \multicolumn{2}{|c|}{ L.S.D. at 0,05 } & $\begin{array}{c}A=1.72 \\
A B=N . S . \\
B C=N . S .\end{array}$ & \multicolumn{3}{|c|}{$\begin{array}{l}B=1.73 \\
A C=\text { N.S. } \\
A B C=\text { N.S. }\end{array}$} & $\begin{array}{l}A=1.55 \\
A B=N \\
B C=N .\end{array}$ & \multicolumn{2}{|c|}{$\begin{array}{c}\mathrm{B}=1.64 \quad \mathrm{C}= \\
\mathrm{AC}=\mathrm{N} . \mathrm{S} . \\
\mathrm{ABC}=\mathrm{N} . \mathrm{S} .\end{array}$} & \\
\hline
\end{tabular}

$\mathrm{N}_{1}=120 \mathrm{~kg} \mathrm{~N} /$ fed as soil application

$\mathrm{N}_{2}=90 \mathrm{~kg} \mathrm{~N} /$ fed as soil application + two time foliar spraying of $2 \%$ urea

$\mathrm{N}_{3}=60 \mathrm{~kg} \mathrm{~N} /$ fed as soil application + two time foliar spraying of $2 \%$ urea

$\mathrm{P}_{1}=13.6 \mathrm{~kg} \mathrm{P} / \mathrm{fed}$ as soil application

$\mathrm{P}_{2}=6.8 \mathrm{~kg} \mathrm{P} / \mathrm{fed}$ as soil application + two time foliar spraying of $0.33 \% \mathrm{P}$

$\mathrm{P}_{3}=$ tow time foliar spraying of $0.33 \% \mathrm{P}$

$\mathrm{K}_{1}=39.9 \mathrm{~kg} \mathrm{~K} / \mathrm{fed}$ as soil application

$\mathrm{K}_{2}=19.9 \mathrm{~kg} \mathrm{~K} / \mathrm{fed}$ as soil application+ two time foliar spraying of $2 \%$ potassium sulphate

$\mathrm{K}_{3}=$ two time foliar spraying of $2 \%$ potassium sulphate 
Table 3. Effect of soil and foliar application of different N,P and K levels on dry weight of 60 days from planting ( g/ plant )

\begin{tabular}{|c|c|c|c|c|c|c|c|c|c|}
\hline \multirow{3}{*}{ Nitrogen } & \multirow{3}{*}{ Phosphorus } & \multicolumn{8}{|c|}{ Potassium } \\
\hline & & \multicolumn{4}{|c|}{ First season } & \multicolumn{4}{|c|}{ second season } \\
\hline & & $\mathbf{K}_{1}$ & $\mathbf{K}_{2}$ & $\mathbf{K}_{3}$ & Mean & $\mathbf{K}_{1}$ & $\mathbf{K}_{2}$ & $\mathbf{K}_{3}$ & Mean \\
\hline \multirow{3}{*}{$\mathbf{N}_{1}$} & $\mathbf{P}_{1}$ & 92.10 & 91.50 & 88.05 & 90.55 & 91.25 & 90.80 & 87.40 & 89.82 \\
\hline & $\mathbf{P}_{2}$ & 91.50 & 90.60 & 87.80 & 89.96 & 89.75 & 89.50 & 86.10 & 88.45 \\
\hline & $\mathbf{P}_{3}$ & 86.70 & 86.50 & 83.30 & 85.50 & 87.00 & 85.30 & 83.50 & 85.27 \\
\hline \multicolumn{2}{|l|}{ Mean } & 90.10 & 89.53 & 86.38 & 88.67 & 89.33 & 88.53 & 85.67 & $\mathbf{8 7 . 8 5}$ \\
\hline \multirow{3}{*}{$\mathbf{N}_{2}$} & $\mathbf{P}_{1}$ & 90.10 & 89.40 & 82.90 & 87.47 & 89.56 & 86.15 & 83.76 & 86.49 \\
\hline & $\mathbf{P}_{2}$ & 89.80 & 89.50 & 82.00 & 87.10 & 88.44 & 85.76 & 82.35 & 85.52 \\
\hline & $\mathbf{P}_{3}$ & 83.10 & 83.00 & 76.77 & 81.62 & 82.65 & 81.12 & 77.37 & 80.38 \\
\hline \multicolumn{2}{|l|}{ Mean } & 87.67 & 87.30 & 80.56 & 85.40 & 86.88 & 84.34 & 81.16 & 84.13 \\
\hline \multirow{3}{*}{$\mathbf{N}_{3}$} & $\mathbf{P}_{1}$ & 75.90 & 75.50 & 70.33 & 73.91 & 78.27 & 77.00 & 75.10 & 76.79 \\
\hline & $\mathbf{P}_{2}$ & 75.15 & 75.10 & 70.10 & 73.45 & 77.25 & 75.25 & 73.50 & 75.33 \\
\hline & $\mathbf{P}_{3}$ & 71.22 & 71.00 & 69.20 & 70.47 & 73.10 & 73.05 & 70.30 & 72.15 \\
\hline \multicolumn{2}{|l|}{ Mean } & 74.09 & 73.87 & 69.88 & 72.61 & 76.21 & 75.10 & 72.97 & 74.76 \\
\hline \multirow{3}{*}{ Mean of $P$} & $\mathbf{P}_{1}$ & 86.03 & 85.47 & 80.43 & 83.98 & 86.36 & 84.65 & 82.09 & 84.37 \\
\hline & $\mathbf{P}_{2}$ & 85.48 & 85.07 & 79.97 & 83.51 & 85.15 & 83.50 & 80.65 & 83.10 \\
\hline & $\mathbf{P}_{3}$ & 80.34 & 80.17 & 76.42 & 78.98 & 80.92 & 79.82 & 77.06 & 79.27 \\
\hline \multicolumn{2}{|l|}{ Mean of $\mathrm{K}$} & 83.95 & 83.57 & 78.94 & 82.15 & 84.14 & 82.66 & 79.93 & 82.24 \\
\hline \multicolumn{2}{|c|}{ L.S.D. at 0,05 } & $\begin{array}{l}\mathrm{A}=3.95 \\
\mathrm{AB}=\mathrm{N} \\
\mathrm{BC}=\mathrm{N}\end{array}$ & \multicolumn{3}{|c|}{$\begin{array}{r}\mathrm{B}=2.01 \mathrm{C} \\
\mathrm{AC}=\mathrm{NS} \\
\mathrm{ABC}=\mathrm{NS}\end{array}$} & $\begin{array}{l}A=3.83 \\
A B=N S \\
B C=N S\end{array}$ & \multicolumn{3}{|c|}{$\begin{array}{c}\mathrm{B}=1.73 \\
\mathrm{AC}=\mathrm{NS} \\
\mathrm{ABC}=\mathrm{NS}\end{array}$} \\
\hline
\end{tabular}

$\mathrm{N}_{1}=120 \mathrm{~kg} \mathrm{~N} /$ fed as soil application

$\mathrm{N}_{2}=90 \mathrm{~kg} \mathrm{~N} /$ fed as soil application + two time foliar spraying of $2 \%$ urea

$\mathrm{N}_{3}=60 \mathrm{~kg} \mathrm{~N} /$ fed as soil application + two time foliar spraying of $2 \%$ urea

$\mathrm{P}_{1}=13.6 \mathrm{~kg} \mathrm{P} / \mathrm{fed}$ as soil application

$\mathrm{P}_{2}=6.8 \mathrm{~kg} \mathrm{P} / \mathrm{fed}$ as soil application + two time foliar spraying of $0.33 \% \mathrm{P}$

$\mathrm{P}_{3}=$ tow time foliar spraying of $0.33 \% \mathrm{P}$

$\mathrm{K}_{1}=39.9 \mathrm{~kg} \mathrm{~K} / \mathrm{fed}$ as soil application

$\mathrm{K}_{2}=19.9 \mathrm{~kg} \mathrm{~K} / \mathrm{fed}$ as soil application+ two time foliar spraying of $2 \%$ potassium sulphate

$\mathrm{K}_{3}=$ two time foliar spraying of $2 \%$ potassium sulphate

Table 4. Effect of soil and foliar application of different N,P and K levels on number of leaves/ plant at of 60 days from plantin

\begin{tabular}{|c|c|c|c|c|c|c|c|c|c|}
\hline \multirow{3}{*}{ Nitrogen } & \multirow{3}{*}{ Phosphorus } & \multicolumn{8}{|c|}{ Potassium } \\
\hline & & \multicolumn{4}{|c|}{ First season } & \multicolumn{4}{|c|}{ second season } \\
\hline & & $\mathbf{K}_{1}$ & $\mathbf{K}_{2}$ & $\mathbf{K}_{3}$ & Mean & $\mathbf{K}_{1}$ & $\mathbf{K}_{2}$ & $\mathbf{K}_{3}$ & Mean \\
\hline \multirow{3}{*}{$\mathbf{N}_{1}$} & $\mathbf{P}_{1}$ & 13.13 & 13.09 & 12.90 & 13.04 & 13.09 & 13.02 & 12.20 & 12.77 \\
\hline & $\mathbf{P}_{2}$ & 13.10 & 13.05 & 12.80 & 12.98 & 13.04 & 13.01 & 12.10 & 12.72 \\
\hline & $\mathbf{P}_{3}$ & 12.80 & 12.65 & 12.20 & 12.55 & 12.71 & 12.68 & 12.00 & 12.46 \\
\hline \multicolumn{2}{|l|}{ Mean } & 13.01 & 12.93 & 12.63 & 12.86 & 12.95 & 12.90 & 12.10 & 12.65 \\
\hline \multirow{3}{*}{$\mathbf{N}_{2}$} & $\mathbf{P}_{1}$ & 13.10 & 13.08 & 12.72 & 12.97 & 13.01 & 12.97 & 12.15 & 12.69 \\
\hline & $\mathbf{P}_{2}$ & 13.03 & 13.00 & 12.70 & 12.91 & 12.97 & 12.95 & 12.09 & 12.67 \\
\hline & $\mathbf{P}_{3}$ & 12.64 & 12.60 & 12.40 & 12.55 & 12.72 & 12.70 & 12.00 & 12.47 \\
\hline \multicolumn{2}{|l|}{ Mean } & 12.92 & 12.89 & 12.61 & 12.81 & 12.90 & 12.87 & 12.08 & 12.61 \\
\hline \multirow{3}{*}{$\mathbf{N}_{3}$} & $\mathbf{P}_{1}$ & 12.60 & 12.60 & 12.34 & 12.51 & 12.70 & 12.69 & 12.10 & 12.50 \\
\hline & $\mathbf{P}_{2}$ & 12.54 & 12.50 & 12.30 & 12.45 & 12.66 & 12.56 & 12.00 & 12.41 \\
\hline & $\mathbf{P}_{3}$ & 12.10 & 12.10 & 11.80 & 12.00 & 12.52 & 12.53 & 11.70 & 12.25 \\
\hline \multirow[t]{2}{*}{ Mean } & & 12.41 & 12.40 & 12.15 & 12.32 & 12.63 & 12.59 & 11.93 & 12.39 \\
\hline & $\mathbf{P}_{1}$ & 12.94 & 12.92 & 12.65 & 12.84 & 12.93 & 12.89 & 12.15 & 12.66 \\
\hline \multirow[t]{2}{*}{ Mean of $\mathbf{P}$} & $\mathbf{P}_{2}$ & 12.89 & 12.85 & 12.60 & 12.78 & 12.89 & 12.84 & 12.06 & 12.60 \\
\hline & $\mathbf{P}_{3}$ & 12.51 & 12.45 & 12.13 & 12.36 & 12.65 & 12.64 & 11.90 & 12.40 \\
\hline \multicolumn{2}{|l|}{ Mean of $\mathrm{K}$} & 12.78 & 12.74 & 12.46 & 12.66 & 12.82 & 12.79 & 12.04 & 12.55 \\
\hline \multicolumn{2}{|c|}{ L.S.D. at 0,05} & $\begin{array}{l}\mathrm{A}=\mathbf{N S} \\
\mathrm{AB}=\mathbf{N S} \\
\mathrm{BC}=\mathbf{N S}\end{array}$ & \multicolumn{2}{|c|}{$\begin{array}{c}\mathrm{B}=\mathrm{NS} \\
\mathrm{AC}=\mathrm{NS} \\
\mathrm{ABC}=\mathrm{NS}\end{array}$} & & $\begin{array}{l}\mathbf{A}=\mathbf{N S} \\
\mathrm{AB}=\mathbf{N S} \\
\mathrm{BC}=\mathbf{N S}\end{array}$ & \multicolumn{2}{|c|}{$\begin{array}{c}\mathrm{B}=\mathrm{NS} \\
\mathrm{AC}=\mathrm{NS} \\
\mathrm{ABC}=\mathrm{NS}\end{array}$} & \\
\hline
\end{tabular}

$\mathrm{N}_{1}=120 \mathrm{~kg} \mathrm{~N} /$ fed as soil application 
$\mathrm{N}_{2}=90 \mathrm{~kg} \mathrm{~N} /$ fed as soil application + two time foliar spraying of $2 \%$ urea

$\mathrm{N}_{3}=60 \mathrm{~kg} \mathrm{~N} /$ fed as soil application + two time foliar spraying of $2 \%$ urea

$\mathrm{P}_{1}=13.6 \mathrm{~kg} \mathrm{P} / \mathrm{fed}$ as soil application

$\mathrm{P}_{2}=6.8 \mathrm{~kg} \mathrm{P} / \mathrm{fed}$ as soil application + two time foliar spraying of $0.33 \% \mathrm{P}$

$\mathrm{P}_{3}=$ tow time foliar spraying of $0.33 \% \mathrm{P}$

$\mathrm{K}_{1}=39.9 \mathrm{~kg} \mathrm{~K} / \mathrm{fed}$ as soil application

$\mathrm{K}_{2}=19.9 \mathrm{~kg} \mathrm{~K} / \mathrm{fed}$ as soil application+ two time foliar spraying of $2 \%$ potassium sulphate

$\mathrm{K}_{3}=$ two time foliar spraying of $2 \%$ potassium sulphate

Table 5. Effect of soil and foliar application of different N,P and $\mathrm{K}$ levels on leaf area of 60 days from planting $\left(\mathrm{cm}^{2}\right)$

\begin{tabular}{|c|c|c|c|c|c|c|c|c|c|}
\hline \multirow{3}{*}{ Nitrogen } & \multirow{3}{*}{ Phosphorus } & \multicolumn{8}{|c|}{ Potassium } \\
\hline & & \multicolumn{4}{|c|}{ First season } & \multicolumn{4}{|c|}{ second season } \\
\hline & & $\mathbf{K}_{1}$ & $\mathbf{K}_{2}$ & $\mathbf{K}_{\mathbf{3}}$ & Mean & $\mathbf{K}_{1}$ & $\mathbf{K}_{2}$ & $\mathbf{K}_{3}$ & Mean \\
\hline \multirow{3}{*}{$\mathbf{N}_{1}$} & $\mathbf{P}_{1}$ & 680.11 & 680.00 & 633.60 & 664.57 & 697.85 & 688.85 & 648.90 & 678.53 \\
\hline & $\mathbf{P}_{2}$ & 670.20 & 671.11 & 630.50 & 657.27 & 680.34 & 680.00 & 647.07 & 667.47 \\
\hline & $\mathbf{P}_{3}$ & 640.30 & 636.12 & 600.22 & 625.55 & 658.65 & 632.02 & 607.89 & 632.85 \\
\hline \multicolumn{2}{|l|}{ Mean } & 663.47 & 662.41 & 621.44 & 649.13 & 669.96 & 666.96 & 632.95 & 659.62 \\
\hline \multirow{3}{*}{$\mathbf{N}_{2}$} & $\mathbf{P}_{1}$ & 651.22 & 650.30 & 600.11 & 633.88 & 634.40 & 630.20 & 604.67 & 623.09 \\
\hline & $\mathbf{P}_{2}$ & 648.00 & 645.70 & 595.20 & 629.63 & 620.70 & 613.13 & 601.25 & 611.69 \\
\hline & $\mathbf{P}_{3}$ & 601.50 & 599.08 & 560.50 & 587.03 & 595.55 & 576.62 & 576.62 & 582.93 \\
\hline \multicolumn{2}{|l|}{ Mean } & 633.57 & 631,69 & 585.27 & 616.85 & 616.88 & 606.65 & 594.18 & 605.90 \\
\hline \multirow{3}{*}{$\mathbf{N}_{3}$} & $\mathbf{P}_{1}$ & 540.11 & 536.02 & 500.30 & 525.48 & 538.90 & 534.10 & 511.40 & 526.47 \\
\hline & $\mathbf{P}_{2}$ & 531.40 & 530.90 & 500.00 & 520.77 & 526.74 & 525.65 & 500.20 & $\mathbf{5 1 7 . 5 3}$ \\
\hline & $\mathbf{P}_{3}$ & 453.11 & 450.20 & 430.20 & 444.50 & 480.11 & 470.20 & 425.40 & 458.57 \\
\hline \multicolumn{2}{|l|}{ Mean } & 508.21 & 505.71 & 476.83 & 496.92 & 513.58 & 510.32 & 479.00 & 500.86 \\
\hline \multirow{3}{*}{ Mean of $P$} & $\mathbf{P}_{1}$ & 623.81 & 622.11 & 578.00 & 607.98 & 622.05 & 617.72 & 588.32 & 609.36 \\
\hline & $\mathbf{P}_{2}$ & 616.53 & 615.90 & 575.23 & 602.56 & 609.26 & 606.59 & 581.17 & 599.01 \\
\hline & $\mathbf{P}_{3}$ & 564.97 & 561.80 & 530.31 & 552.36 & 578.10 & 559.61 & 536.64 & 558.12 \\
\hline \multicolumn{2}{|l|}{ Mean of K } & 601.77 & 599.94 & 561.18 & 587.63 & 603.14 & 594.64 & 568.71 & 588.83 \\
\hline \multicolumn{2}{|c|}{ L.S.D. at 0,05} & $\begin{array}{l}\mathrm{A}=11.53 \\
\mathrm{AB}=\mathrm{NS} \\
\mathrm{BC}=\mathrm{NS}\end{array}$ & \multicolumn{3}{|c|}{$\begin{array}{cc}\mathrm{B}=9.16 & \mathrm{C}=8.6 \\
\mathrm{AC}=\mathrm{NS} & \\
\mathrm{ABC}=\mathrm{NS} & \end{array}$} & $\begin{array}{l}\mathrm{A}=\mathbf{1 2 . 0 7} \\
\mathrm{AB}=\mathbf{N S} \\
\mathrm{BC}=\mathbf{N S}\end{array}$ & $\begin{array}{l}\mathrm{B}=12 \\
\mathrm{AC}=1 \\
\mathrm{ABC}\end{array}$ & $\begin{array}{l}03 \mathrm{C}=1 \\
\mathrm{~S} \\
\text { NS }\end{array}$ & 76 \\
\hline
\end{tabular}

$\mathrm{N}_{1}=120 \mathrm{~kg} \mathrm{~N} /$ fed as soil application

$\mathrm{N}_{2}=90 \mathrm{~kg} \mathrm{~N} /$ fed as soil application + two time foliar spraying of $2 \%$ urea

$\mathrm{N}_{3}=60 \mathrm{~kg} \mathrm{~N} /$ fed as soil application + two time foliar spraying of $2 \%$ urea

$\mathrm{P}_{1}=13.6 \mathrm{~kg} \mathrm{P} / \mathrm{fed}$ as soil application

$\mathrm{P}_{2}=6.8 \mathrm{~kg} \mathrm{P} / \mathrm{fed}$ as soil application + two time foliar spraying of $0.33 \% \mathrm{P}$

$\mathrm{P}_{3}=$ tow time foliar spraying of $0.33 \% \mathrm{P}$

$\mathrm{K}_{1}=39.9 \mathrm{~kg} \mathrm{~K} / \mathrm{fed}$ as soil application

$\mathrm{K}_{2}=19.9 \mathrm{~kg} \mathrm{~K} / \mathrm{fed}$ as soil application+ two time foliar spraying of $2 \%$ potassium sulphate

$\mathrm{K}_{3}=$ two time foliar spraying of $2 \%$ potassium sulphate

\section{Yield components}

The present results in Tables 6,7 and 8 indicate that the studied yield components of maize , namely , number of rows/ ear, number of grains / ear and $100-$ grain yield were affected by nitrogen phosphorus and potassium treatments. Statistically, $\mathrm{N}_{1}, \mathrm{P}_{1}$ and $\mathrm{K}_{1}$ treatments gave highest number of rows/ear (12.87, 12.84 and 12.85), number of grains/ear (46.11, 45.45 and 45.44) and 100-grain weight (29.67, 29.48 and 29.41), equal to those obtained under $\mathrm{N}_{1}(12.81,45.44$ and 29.36), $\mathrm{P}_{2}$ (12.81, 45.17 and 29.22) and $\mathrm{K}_{2}$ (12.82, 45.25 and 29.14) respectively in the first season. The same trend was obtained in the second season. Such improvement in yield components is mainly due to the effects of the applied the nutrient on maize growth as
Soil application and foliar spray to gather. These results are in line with those obtained by Salwau and Shams El-Din (1992) and Gebraiel et al (2005) for nitrogen; El- Azab (2015) and Gul et al (2015) for phosphorus and Mqsood et al (2013) for potassium treatments.

Concerning the effect of the interaction between treatment on maize yield components, in general the data reveal that the highest number of rows/ ear , number of grains/ ear and 100 - grain weight were obtained dromr maize plants treated with $\mathrm{N}_{1}$ or $\mathrm{N}_{2}+$ $\mathrm{P}_{1}$ or $\mathrm{P}_{2}+\mathrm{K}_{1}$ or $\mathrm{K}_{2}$ treatment. On the other hand the plants supplied with $\mathrm{N}_{3}+\mathrm{P}_{3}+\mathrm{K}_{3}$ treatment yielded the lowest maize yield components. As compared with other two previous treatments. 
Table 6. Effect of soil and foliar application of different N,P and K levels on number of rows / ear

\begin{tabular}{|c|c|c|c|c|c|c|c|c|c|}
\hline \multirow{3}{*}{ Nitrogen } & \multirow{3}{*}{ Phosphorus } & \multicolumn{8}{|c|}{ Potassium } \\
\hline & & \multicolumn{4}{|c|}{ First season } & \multicolumn{4}{|c|}{ second season } \\
\hline & & $\mathbf{K}_{1}$ & $\mathbf{K}_{2}$ & $\mathbf{K}_{3}$ & Mean & $K_{1}$ & $\mathbf{K}_{2}$ & $\mathbf{K}_{3}$ & Mean \\
\hline \multirow{3}{*}{$\mathbf{N}_{1}$} & $\mathbf{P}_{1}$ & 13.00 & 12.95 & 12.81 & 12.92 & 13.05 & 13.00 & 12.88 & 12.98 \\
\hline & $\mathbf{P}_{2}$ & 12.91 & 12.90 & 12.81 & 12.87 & 12.90 & 12.90 & 12.85 & 12.88 \\
\hline & $\mathbf{P}_{3}$ & 12.87 & 12.83 & 12.79 & 12.83 & 12.80 & 12.85 & 12.80 & 12.82 \\
\hline Mean & & 12.92 & 12.89 & 12.80 & 12.87 & 12.92 & 12.92 & 12.84 & 12.89 \\
\hline \multirow{3}{*}{$\mathbf{N}_{2}$} & $\mathbf{P}_{1}$ & 12.92 & 12.90 & 12.76 & 12.86 & 12.95 & 12.90 & 12.80 & 12.88 \\
\hline & $\mathbf{P}_{2}$ & 12.92 & 12.85 & 12.75 & 12.84 & 12.91 & 12.86 & 12.80 & 12.86 \\
\hline & $\mathbf{P}_{3}$ & 12.76 & 12.72 & 12.70 & 12.73 & 12.83 & 12.80 & 12.75 & 12.79 \\
\hline Mean & & 12.87 & 12.82 & 12.74 & 12.81 & 12.90 & 12.85 & 12.78 & 12.84 \\
\hline \multirow{3}{*}{$\mathbf{N}_{3}$} & $\mathbf{P}_{1}$ & 12.81 & 12.78 & 12.66 & 12.75 & 12.80 & 12.80 & 12.70 & 12.77 \\
\hline & $\mathbf{P}_{2}$ & 12.76 & 12.73 & 12.66 & 12.72 & 12.80 & 12.75 & 12.66 & 12.73 \\
\hline & $\mathbf{P}_{3}$ & 12.71 & 12.68 & 12.51 & 12.63 & 12.70 & 12.63 & 12.50 & 12.61 \\
\hline Mean & & 12.76 & 12.73 & 12.61 & 12.70 & 12.76 & 12.73 & 12.62 & 12.70 \\
\hline \multirow{3}{*}{ Mean of $P$} & $\mathbf{P}_{1}$ & 12.91 & 12.88 & 12.74 & 12.84 & 12.93 & 12.90 & 12.79 & 12.88 \\
\hline & $\mathbf{P}_{2}$ & 12.86 & 12.83 & 12.74 & 12.81 & 12.86 & 12.84 & 12.77 & 12.82 \\
\hline & $\mathbf{P}_{3}$ & 12.78 & 12.74 & 12.67 & 12.73 & 12.78 & 12.76 & 12.68 & 12.74 \\
\hline \multicolumn{2}{|l|}{ Mean of K } & 12.85 & 12.82 & 12.72 & 12.79 & 12.86 & 12.83 & 12.75 & 12.81 \\
\hline \multicolumn{2}{|c|}{ L.S.D. at $\mathbf{0 , 0 5}$} & $\begin{array}{l}\mathrm{A}=\mathbf{0 . 0 8} \\
\mathrm{AB}=\mathrm{NS} \\
\mathrm{BC}=\mathrm{NS}\end{array}$ & \multicolumn{3}{|c|}{$\begin{array}{l}\mathrm{B}=0.09 \quad \mathrm{C}=0.07 \\
\mathrm{AC}=\mathrm{NS} \\
\mathrm{ABC}=\mathrm{NS}\end{array}$} & $\begin{array}{l}\mathrm{A}=0.07 \\
\mathrm{AB}=\mathrm{NS} \\
\mathrm{BC}=\mathrm{NS}\end{array}$ & $\begin{array}{l}B=C \\
A C= \\
A B C\end{array}$ & \multicolumn{2}{|c|}{$\begin{array}{r}C=0.06 \\
S\end{array}$} \\
\hline
\end{tabular}

$\mathrm{N}_{1}=120 \mathrm{~kg} \mathrm{~N} /$ fed as soil application

$\mathrm{N}_{2}=90 \mathrm{~kg} \mathrm{~N} /$ fed as soil application + two time foliar spraying of $2 \%$ urea

$\mathrm{N}_{3}=60 \mathrm{~kg} \mathrm{~N} /$ fed as soil application + two time foliar spraying of $2 \%$ urea

$\mathrm{P}_{1}=13.6 \mathrm{~kg} \mathrm{P} / \mathrm{fed}$ as soil application

$\mathrm{P}_{2}=6.8 \mathrm{~kg} \mathrm{P} / \mathrm{fed}$ as soil application + two time foliar spraying of $0.33 \% \mathrm{P}$

$\mathrm{P}_{3}=$ tow time foliar spraying of $0.33 \% \mathrm{P}$

$\mathrm{K}_{1}=39.9 \mathrm{~kg} \mathrm{~K} /$ fed as soil application

$\mathrm{K}_{2}=19.9 \mathrm{~kg} \mathrm{~K} / \mathrm{fed}$ as soil application+ two time foliar spraying of $2 \%$ potassium sulphate

$\mathrm{K}_{3}=$ two time foliar spraying of $2 \%$ potassium sulphate

Table 7. Effect of soil and foliar application of different N,P and K levels on number of grains / row

\begin{tabular}{|c|c|c|c|c|c|c|c|c|c|}
\hline \multirow{3}{*}{ Nitrogen } & \multirow{3}{*}{ Phosphorus } & \multicolumn{8}{|c|}{ Potassium } \\
\hline & & \multicolumn{4}{|c|}{ First season } & \multicolumn{4}{|c|}{ second season } \\
\hline & & $\mathbf{K}_{1}$ & $\mathbf{K}_{2}$ & $\mathbf{K}_{3}$ & Mean & $\mathbf{K}_{1}$ & $\mathbf{K}_{2}$ & $\mathbf{K}_{3}$ & Mean \\
\hline \multirow{3}{*}{$\mathbf{N}_{1}$} & $\mathbf{P}_{1}$ & 46.80 & 46.65 & 45.75 & 46.40 & 46.91 & 46.80 & 45.80 & 46.51 \\
\hline & $\mathbf{P}_{2}$ & 46.70 & 46.53 & 45.60 & 46.28 & 46.85 & 46.73 & 45.69 & 46.42 \\
\hline & $\mathbf{P}_{3}$ & 45.80 & 45.60 & 45.55 & 45.65 & 46.39 & 46.30 & 45.50 & 46.06 \\
\hline \multicolumn{2}{|l|}{ Mean } & 46.43 & 46.26 & 45.63 & 46.11 & 45.72 & 46.61 & 45.67 & 46.33 \\
\hline \multirow{3}{*}{$\mathbf{N}_{2}$} & $\mathbf{P}_{1}$ & 46.11 & 45.90 & 45.20 & 45.74 & 46.09 & 45.87 & 45.30 & 45.75 \\
\hline & $\mathbf{P}_{2}$ & 45.80 & 45.71 & 45.10 & 45.54 & 45.79 & 45.73 & 45.20 & 45.57 \\
\hline & $\mathbf{P}_{3}$ & 45.30 & 45.11 & 44.70 & 45.04 & 45.69 & 45.32 & 44.55 & 45.19 \\
\hline \multicolumn{2}{|l|}{ Mean } & 45.75 & 45.57 & 45.00 & 45.44 & 45.86 & 45.64 & 45.02 & 45.51 \\
\hline \multirow{3}{*}{$\mathbf{N}_{3}$} & $\mathbf{P}_{1}$ & 44.55 & 44.30 & 43.80 & 44.22 & 44.66 & 43.69 & 42.51 & 43.62 \\
\hline & $\mathbf{P}_{2}$ & 44.10 & 43.90 & 43.05 & 43.68 & 43.52 & 43.35 & 41.64 & 42.84 \\
\hline & $\mathbf{P}_{3}$ & 43.80 & 43.50 & 42.80 & 43.37 & 43.05 & 42.92 & 41.45 & 42.47 \\
\hline \multicolumn{2}{|l|}{ Mean } & 44.15 & 43.90 & 43.22 & 43.76 & 43.75 & 43.32 & 41.87 & 42.98 \\
\hline \multirow{3}{*}{ Mean of $P$} & $P_{1}$ & 45.82 & 45.62 & 44.92 & 45.45 & 45.89 & 45.45 & 44.54 & 45.29 \\
\hline & $P_{2}$ & 44.53 & 45.38 & 44.58 & 45.17 & 45.39 & 45.27 & 44.18 & 44.94 \\
\hline & $P_{3}$ & 44.97 & 44.74 & 44.35 & 44.69 & 45.04 & 44.85 & 43.83 & 44.57 \\
\hline \multicolumn{2}{|l|}{ Mean of K } & 45.44 & 45.25 & 44.62 & 45.10 & 45.44 & 45.19 & 44.18 & 44.92 \\
\hline \multicolumn{2}{|c|}{ L.S.D. at 0,05} & $\begin{array}{l}\mathrm{A}=\mathbf{0 . 9 3} \\
\mathrm{AB}=\mathrm{NS} \\
\mathrm{BC}=\mathrm{NS}\end{array}$ & $\begin{array}{r}\mathbf{B}=\mathbf{0} \\
\mathbf{A C}=1 \\
\mathrm{ABC}=\end{array}$ & $3 \mathrm{C}=$ & & $\begin{array}{l}\mathrm{A}=\mathbf{0 . 9 7} \\
\mathrm{AB}=\mathrm{NS} \\
\mathrm{BC}=\mathrm{NS}\end{array}$ & $\begin{array}{r}\mathrm{B}=0 \\
\mathrm{AC}= \\
\mathrm{ABC}=\end{array}$ & $\begin{array}{l}S \\
S\end{array}$ & \\
\hline
\end{tabular}

$\mathrm{N}_{1}=120 \mathrm{~kg} \mathrm{~N} /$ fed as soil application

$\mathrm{N}_{2}=90 \mathrm{~kg} \mathrm{~N} /$ fed as soil application + two time foliar spraying of $2 \%$ urea

$\mathrm{N}_{3}=60 \mathrm{~kg} \mathrm{~N} /$ fed as soil application + two time foliar spraying of $2 \%$ urea 
$\mathrm{P}_{1}=13.6 \mathrm{~kg} \mathrm{P} / \mathrm{fed}$ as soil application

$\mathrm{P}_{2}=6.8 \mathrm{~kg} \mathrm{P} / \mathrm{fed}$ as soil application + two time foliar spraying of $0.33 \% \mathrm{P}$

$\mathrm{P}_{3}=$ tow time foliar spraying of $0.33 \% \mathrm{P}$

$\mathrm{K}_{1}=39.9 \mathrm{~kg} \mathrm{~K} / \mathrm{fed}$ as soil application

$\mathrm{K}_{2}=19.9 \mathrm{~kg} \mathrm{~K} / \mathrm{fed}$ as soil application+ two time foliar spraying of $2 \%$ potassium sulphate

$\mathrm{K}_{3}=$ two time foliar spraying of $2 \%$ potassium sulphate

Table 8. Effect of soil and foliar application of different N,P and K levels on 100 - grain weight ( $g$ )

\begin{tabular}{|c|c|c|c|c|c|c|c|c|c|}
\hline \multirow{3}{*}{ Nitrogen } & \multirow{3}{*}{ Phosphorus } & \multicolumn{8}{|c|}{ Potassium } \\
\hline & & \multicolumn{4}{|c|}{ First season } & \multicolumn{4}{|c|}{ second season } \\
\hline & & $K_{1}$ & $\mathbf{K}_{2}$ & $\mathbf{K}_{3}$ & Mean & $\mathbf{K}_{1}$ & $\mathbf{K}_{2}$ & $\mathbf{K}_{3}$ & Mean \\
\hline \multirow{3}{*}{$\mathbf{N}_{1}$} & $\mathbf{P}_{1}$ & 30.40 & 30.10 & 29.80 & 30.10 & 30.90 & 30.76 & 30.60 & 30.75 \\
\hline & $\mathbf{P}_{2}$ & 30.10 & 29.80 & 29.50 & 29.80 & 30.77 & 30.69 & 30.30 & 30.59 \\
\hline & $\mathbf{P}_{3}$ & 29.40 & 29.10 & 28.80 & 29.10 & 30.56 & 30.37 & 30.05 & 30.33 \\
\hline Mean & & 29.97 & 29.67 & 29.37 & 29.67 & 30.74 & 30.61 & 30.32 & 30.56 \\
\hline \multirow{3}{*}{$\mathbf{N}_{2}$} & $\mathbf{P}_{1}$ & 30.15 & 30.00 & 29.30 & 29.82 & 30.37 & 29.75 & 29.11 & 29.74 \\
\hline & $\mathbf{P}_{2}$ & 29.90 & 29.70 & 29.10 & 29.57 & 29.90 & 29.60 & 28.80 & 29.43 \\
\hline & $\mathbf{P}_{3}$ & 29.10 & 28.80 & 28.20 & 28.70 & 29.10 & 28.90 & 28.60 & 28.87 \\
\hline Mean & & 29.72 & 29.50 & 28.87 & 29.36 & 29.79 & 29.42 & 28.84 & 29.35 \\
\hline \multirow{3}{*}{$\mathbf{N}_{3}$} & $\mathbf{P}_{1}$ & 28.90 & 28.60 & 28.05 & 28.52 & 29.05 & 28.85 & 28.69 & 28.86 \\
\hline & $\mathbf{P}_{2}$ & 28.70 & 28.50 & 27.70 & 28.30 & 28.77 & 28.50 & 27.90 & 28.39 \\
\hline & $\mathbf{P}_{3}$ & 28.00 & 27.70 & 27.05 & 27.58 & 28.10 & 27.80 & 26.61 & 27.50 \\
\hline Mean & & 28.53 & 28.27 & 27.60 & 28.13 & 28.64 & 28.38 & 27.73 & 28.25 \\
\hline \multirow{3}{*}{ Mean of $P$} & $P_{1}$ & 29.82 & 29.57 & 29.05 & 29.48 & 30.11 & 29.79 & 29.47 & 29.79 \\
\hline & $P_{2}$ & 29.57 & 29.33 & 28.77 & 29.22 & 29.81 & 29.60 & 29.00 & 29.47 \\
\hline & $P_{3}$ & 28.85 & 28.53 & 28.02 & 28.46 & 29.25 & 29.02 & 28.85 & 29.04 \\
\hline \multicolumn{2}{|l|}{ Mean of K } & 29.41 & 29.14 & 28.61 & 29.05 & 29.72 & 29.49 & 29.11 & 29.43 \\
\hline \multicolumn{2}{|c|}{ L.S.D. at $\mathbf{0 , 0 5}$} & $\begin{array}{l}\mathrm{A}=0.3 \\
\mathrm{AB}=\mathrm{N} \\
\mathrm{BC}=\mathrm{N}\end{array}$ & $\begin{array}{l}\mathrm{B}= \\
\mathrm{AC} \\
\mathrm{ABC}\end{array}$ & $\begin{array}{l}30 \mathrm{C}= \\
\mathrm{NS} \\
=\mathrm{NS}\end{array}$ & & $\begin{array}{l}A=0.39 \\
A B=N S \\
B C=N S\end{array}$ & $\begin{array}{r}\mathbf{B}= \\
\mathrm{AC} \\
\mathrm{ABC} \\
\end{array}$ & $\begin{array}{l}33 \quad \mathrm{C}= \\
\text { NS } \\
\text { NS }\end{array}$ & \\
\hline
\end{tabular}

$\mathrm{N}_{1}=120 \mathrm{~kg} \mathrm{~N} /$ fed as soil application

$\mathrm{N}_{2}=90 \mathrm{~kg} \mathrm{~N} /$ fed as soil application + two time foliar spraying of $2 \%$ urea

$\mathrm{N}_{3}=60 \mathrm{~kg} \mathrm{~N} /$ fed as soil application + two time foliar spraying of $2 \%$ urea

$\mathrm{P}_{1}=13.6 \mathrm{~kg} \mathrm{P} / \mathrm{fed}$ as soil application

$\mathrm{P}_{2}=6.8 \mathrm{~kg} \mathrm{P} / \mathrm{fed}$ as soil application + two time foliar spraying of $0.33 \% \mathrm{P}$

$\mathrm{P}_{3}=$ tow time foliar spraying of $0.33 \% \mathrm{P}$

$\mathrm{K}_{1}=39.9 \mathrm{~kg} \mathrm{~K} / \mathrm{fed}$ as soil application

$\mathrm{K}_{2}=19.9 \mathrm{~kg} \mathrm{~K} / \mathrm{fed}$ as soil application+ two time foliar spraying of $2 \%$ potassium sulphate

$\mathrm{K}_{3}=$ two time foliar spraying of $2 \%$ potassium sulphate

\section{Yield}

The data listed in Table 9, 10 and 11 represent the response of maize yields , i.e., grains, stover and biological yields to the NPK fertilization. It is evident from the data that maize yields were significantly responded to nitrogen, phosphorus and potassium fertilization in both seasons. The highest maize yields was recorded for the plants fertilized with $\mathrm{N}_{1}+\mathrm{P}_{1}+$ $\mathrm{K}_{1}$. On the other hand $\mathrm{N}_{3}+\mathrm{P}_{3}+\mathrm{K}_{3}$ treatments produced the lowest maize yield. The relative increasing in grain yield due to $\mathrm{N}_{1}$ and $\mathrm{N}_{2}, \mathrm{P}_{1}$ or $\mathrm{P}_{2}$ and $K_{1}$ or $K_{2}$ was 40.3 or $40.2 ; 30.0$ or 29.9 ; and 23.4 or $23.3 \%$ over $\mathrm{N}_{3}$ treatment in the first season, respectively. The corresponding increase in the second season was 35.4 or $33.7 ; 26.3$ or $24.6 \%$ and 19.6 or $18.9 \%$ in the abovementioned order. Similer trends were obtained for stover and biological yields. These results could be explained by effects of these treatments on growth and yield components of maize as mentioned before. These results are similar to those obtained by Allam et al (2001) and Ismail et al (2006).
Regarding the interaction between treatments or among them, the results clearly reveal that grains, stover and biological yields were not significantly affected by these interaction. This means that the highest values of grains and stover yields were exhibited under $\mathrm{N}_{1}+\mathrm{P}_{1}+\mathrm{K}_{1}$ in both seasons except for grain yield in the first season, where the treatment of $\mathrm{N} 1+\mathrm{P} 2+\mathrm{K} 1$ gave the highest value. On the other hand, $\mathrm{N}_{3}+\mathrm{P}_{3}+\mathrm{K}_{3}$ treatment recorded the lowest values of grains, stover and biological yields.

\section{Net income}

Data in Table 12 represent the response of maize grain net income to NPK fertilization. The data indicate that $\mathrm{N}_{2}, \mathrm{P}_{1}$ or $\mathrm{P}_{2}$ as well as $\mathrm{K}_{1}$ or $\mathrm{K}_{2}$ gave the highest net income of maize grains, while $\mathrm{N}_{3}, \mathrm{P}_{3}$ or $\mathrm{K}_{3}$ recorded the lowest net income in the first seasons and second seasons. The value of net income resulted from $\mathrm{N}_{2}, \mathrm{P}_{1}$ or $\mathrm{P}_{2}$ and $\mathrm{K}_{1}$ or $\mathrm{K}_{2}$ exceeded that obtained from $\mathrm{N}_{3}, \mathrm{P}_{3}$ or $\mathrm{K}_{3}$ by about $2154.86,1356.33$ or 1433.9 and 878.57 or 1195.66 L.E in the first season, respectively. The corresponding values for the second 
season was $1729.86,1408.09$ or 1398.23 and 723.89 or 835.03 in abovementioned order.

Concerning the effect of the interaction between treatments, the results show that the maize plants fertilized $\mathrm{N}_{2}+\mathrm{P}_{2}+\mathrm{K}_{2}$ treatment recorded the highest net income of maize grains, whereas the lowest net income were produced for plants fertilized with $\mathrm{N}_{3}+$ $\mathrm{P}_{3}+\mathrm{K}_{1}$ in the first season and $\mathrm{N}_{2}+\mathrm{P}_{3}+\mathrm{K}_{3}$ in second seasons

Table 9. Effect of soil and foliar application of different N,P and K levels on grain yield $\left(\mathrm{Mg} / \mathrm{h}^{-1}\right)$

\begin{tabular}{|c|c|c|c|c|c|c|c|c|c|}
\hline \multirow{3}{*}{ Nitrogen } & \multirow{3}{*}{ Phosphorus } & \multicolumn{8}{|c|}{ Potassium } \\
\hline & & \multicolumn{4}{|c|}{ First season } & \multicolumn{4}{|c|}{ second season } \\
\hline & & $\mathbf{K}_{1}$ & $\mathbf{K}_{2}$ & $\mathbf{K}_{3}$ & Mean & $\mathbf{K}_{1}$ & $\mathbf{K}_{2}$ & $\mathbf{K}_{3}$ & Mean \\
\hline \multirow{3}{*}{$\mathbf{N}_{1}$} & $\mathbf{P}_{1}$ & 7.217 & 7.190 & 5.719 & 6.709 & 7.340 & 7.267 & 6.100 & 6.902 \\
\hline & $\mathbf{P}_{2}$ & 7.233 & 7.231 & 5.686 & 6.717 & 7.300 & 7.200 & 5.933 & 6.811 \\
\hline & $\mathbf{P}_{3}$ & 5.381 & 5.407 & 4.640 & 5.143 & 5.683 & 5.664 & 5.017 & 5.455 \\
\hline \multicolumn{2}{|l|}{ Mean } & 6.610 & 6.610 & 5.348 & 6.189 & 6.774 & 6.710 & 5.683 & 6.389 \\
\hline \multirow{3}{*}{$\mathbf{N}_{2}$} & $\mathbf{P}_{1}$ & 7.183 & 7.202 & 5.736 & 6.707 & 7.133 & 7.110 & 6.000 & 6.748 \\
\hline & $\mathbf{P}_{2}$ & 7.219 & 7.198 & 5.686 & 6.701 & 7.167 & 7.083 & 5.833 & 6.694 \\
\hline & $\mathbf{P}_{3}$ & 5.374 & 5.350 & 4.707 & 5.144 & 5.740 & 5.733 & 4.867 & 5.447 \\
\hline \multicolumn{2}{|l|}{ Mean } & 6.593 & 6.583 & 5.376 & 6.184 & 6.667 & 6.643 & 5.567 & 6.292 \\
\hline \multirow{3}{*}{$\mathbf{N}_{3}$} & $\mathbf{P}_{1}$ & 5.236 & 5.231 & 3.840 & 4.769 & 5.433 & 5.407 & 4.567 & 5.136 \\
\hline & $\mathbf{P}_{2}$ & 5.219 & 5.200 & 3.857 & 4.759 & 5.417 & 5.400 & 4.267 & 5.028 \\
\hline & $\mathbf{P}_{3}$ & 3.702 & 3.705 & 3.702 & 3.703 & 4.133 & 4.110 & 3.683 & 3.975 \\
\hline \multicolumn{2}{|l|}{ Mean } & 4.719 & 4.712 & 3.800 & 4.410 & 4.995 & 4.971 & 4.171 & 4.713 \\
\hline \multirow{3}{*}{ Mean of $P$} & $\mathbf{P}_{1}$ & 6.545 & 6.540 & 5.098 & 6.061 & 6.636 & 6.595 & 5.555 & 6.262 \\
\hline & $\mathbf{P}_{2}$ & 6.557 & 6.543 & 5.076 & 6.059 & 6.629 & 6.562 & 5.345 & 6.179 \\
\hline & $\mathbf{P}_{3}$ & 4.819 & 4.821 & 4.350 & 4.663 & 5.186 & 5.169 & 4.521 & 4.959 \\
\hline \multicolumn{2}{|l|}{ Mean of K } & 5.974 & 5.969 & 4.840 & 5.594 & 6.150 & 6.110 & 5.140 & 5.800 \\
\hline \multicolumn{2}{|c|}{ L.S.D. at 0,05 } & $\begin{array}{l}A=0.1 \\
A B=N \\
B C=N\end{array}$ & $\begin{array}{c}\mathbf{B}= \\
\mathrm{AC} \\
\mathrm{ABC}\end{array}$ & \multicolumn{2}{|c|}{$2 \mathrm{C}=0.09$} & $\begin{array}{l}\mathbf{A}=0 . \\
\mathbf{A B}=1 \\
\mathbf{B C}=1\end{array}$ & $\begin{array}{c}\mathbf{B}= \\
\mathbf{A C} \\
\mathbf{A B C}\end{array}$ & \multicolumn{2}{|c|}{$3 \mathrm{C}=0.07$} \\
\hline
\end{tabular}

$\mathrm{N}_{1}=120 \mathrm{~kg} \mathrm{~N} /$ fed as soil application

$\mathrm{N}_{2}=90 \mathrm{~kg} \mathrm{~N} /$ fed as soil application + two time foliar spraying of $2 \%$ urea

$\mathrm{N}_{3}=60 \mathrm{~kg} \mathrm{~N} /$ fed as soil application + two time foliar spraying of $2 \%$ urea

$\mathrm{P}_{1}=13.6 \mathrm{~kg} \mathrm{P} / \mathrm{fed}$ as soil application

$\mathrm{P}_{2}=6.8 \mathrm{~kg} \mathrm{P} / \mathrm{fed}$ as soil application + two time foliar spraying of $0.33 \% \mathrm{P}$

$\mathrm{P}_{3}=$ tow time foliar spraying of $0.33 \% \mathrm{P}$

$\mathrm{K}_{1}=39.9 \mathrm{~kg} \mathrm{~K} / \mathrm{fed}$ as soil application

$\mathrm{K}_{2}=19.9 \mathrm{~kg} \mathrm{~K} /$ fed as soil application+ two time foliar spraying of $2 \%$ potassium sulphate

$\mathrm{K}_{3}=$ two time foliar spraying of $2 \%$ potassium sulphate 
$\underline{\text { Table 10. Effect of soil and foliar application of different N,P and K levels on Stover yield }\left(\mathrm{Mg} / \mathrm{h}^{-1}\right)}$

\begin{tabular}{|c|c|c|c|c|c|c|c|c|c|}
\hline \multirow{3}{*}{ Nitrogen } & \multirow{3}{*}{ Phosphorus } & \multicolumn{8}{|c|}{ Potassium } \\
\hline & & \multicolumn{4}{|c|}{ First season } & \multicolumn{4}{|c|}{ second season } \\
\hline & & $\mathbf{K}_{1}$ & $\mathbf{K}_{2}$ & $\mathbf{K}_{3}$ & Mean & $\mathbf{K}_{1}$ & $\mathbf{K}_{2}$ & $\mathbf{K}_{3}$ & Mean \\
\hline \multirow{3}{*}{$\mathbf{N}_{1}$} & $\mathbf{P}_{1}$ & 8.833 & 8.548 & 7.905 & 8.429 & 9.190 & 8.929 & 8.333 & 8.817 \\
\hline & $\mathbf{P}_{2}$ & 8.571 & 8.429 & $\mathbf{7 . 8 3 3}$ & 8.278 & 9.048 & 8.810 & 8.095 & 8.651 \\
\hline & $\mathbf{P}_{3}$ & 8.333 & 8.119 & 7.476 & 7.976 & 8.762 & 8.286 & 7.405 & 8.151 \\
\hline \multicolumn{2}{|l|}{ Mean } & 8.571 & 8.357 & 7.738 & 8.222 & 9.000 & 8.667 & 7.952 & 8.540 \\
\hline \multirow{3}{*}{$\mathbf{N}_{2}$} & $\mathbf{P}_{1}$ & 8.548 & 8.452 & 7.833 & 8.278 & 8.929 & 8.690 & 8.095 & 8.571 \\
\hline & $\mathbf{P}_{2}$ & 8.405 & 8.357 & 7.619 & 8.127 & 8.810 & 8.571 & 8.000 & 8.460 \\
\hline & $\mathbf{P}_{3}$ & 7.952 & 7.857 & 7.262 & 7.690 & 8.333 & 8.286 & 7.786 & 8.135 \\
\hline \multicolumn{2}{|l|}{ Mean } & 8.286 & 8.214 & 7.571 & 8.024 & 8.690 & 8.524 & 7.952 & 8.389 \\
\hline \multirow{3}{*}{$\mathbf{N}_{3}$} & $\mathbf{P}_{1}$ & 7.381 & 7.262 & 6.905 & 7.183 & 7.619 & 7.452 & 6.905 & 7.325 \\
\hline & $\mathbf{P}_{2}$ & 7.143 & 7.000 & 6.690 & 6.944 & 7.381 & 7.262 & 6.190 & 6.944 \\
\hline & $\mathbf{P}_{3}$ & 6.714 & 6.429 & 6.024 & 6.389 & 6.762 & 6.333 & 5.524 & 6.206 \\
\hline \multicolumn{2}{|l|}{ Mean } & 7.071 & 6.905 & 6.548 & 6.841 & 7.262 & 7.024 & 6.238 & 6.841 \\
\hline \multirow{3}{*}{ Mean of $P$} & $\mathbf{P}_{1}$ & 8.262 & 8.095 & 7.548 & 7.968 & 8.571 & 8.357 & 7.786 & 8.238 \\
\hline & $\mathbf{P}_{2}$ & 8.048 & 7.929 & 7.381 & 7.786 & 8.405 & 8.214 & 7.429 & 8.016 \\
\hline & $\mathbf{P}_{3}$ & 7.667 & 7.476 & 6.905 & 7.349 & 7.952 & 7.643 & 6.905 & 7.500 \\
\hline \multicolumn{2}{|l|}{ Mean of $\mathrm{K}$} & 8.000 & 7.833 & 7.286 & 7.706 & 8.310 & 8.071 & 7.381 & 7.921 \\
\hline \multicolumn{2}{|c|}{ L.S.D. at 0,05 } & $\begin{array}{l}A=0.18 \\
A B=N S \\
B C=N S\end{array}$ & $\begin{array}{c}\mathbf{B}=\mathbf{0} \\
\mathrm{AC}= \\
\mathrm{ABC}\end{array}$ & \multicolumn{2}{|c|}{$C=0.16$} & $\begin{array}{l}\mathrm{A}=\mathbf{0 . 3 7} \\
\mathrm{AB}=\mathrm{NS} \\
\mathrm{BC}=\mathrm{NS}\end{array}$ & $\begin{array}{c}\mathbf{B}=0 \\
\mathrm{AC}= \\
\mathrm{ABC}\end{array}$ & \multicolumn{2}{|c|}{$\mathrm{C}=0.22$} \\
\hline
\end{tabular}

$\mathrm{N}_{1}=120 \mathrm{~kg} \mathrm{~N} /$ fed as soil application

$\mathrm{N}_{2}=90 \mathrm{~kg} \mathrm{~N} /$ fed as soil application + two time foliar spraying of $2 \%$ urea

$\mathrm{N}_{3}=60 \mathrm{~kg} \mathrm{~N} /$ fed as soil application + two time foliar spraying of $2 \%$ urea

$\mathrm{P}_{1}=13.6 \mathrm{~kg} \mathrm{P} / \mathrm{fed}$ as soil application

$\mathrm{P}_{2}=6.8 \mathrm{~kg} \mathrm{P} / \mathrm{fed}$ as soil application + two time foliar spraying of $0.33 \% \mathrm{P}$

$\mathrm{P}_{3}=$ tow time foliar spraying of $0.33 \% \mathrm{P}$

$\mathrm{K}_{1}=39.9 \mathrm{~kg} \mathrm{~K} / \mathrm{fed}$ as soil application

$\mathrm{K}_{2}=19.9 \mathrm{~kg} \mathrm{~K} /$ fed as soil application+ two time foliar spraying of $2 \%$ potassium sulphate

$\mathrm{K}_{3}=$ two time foliar spraying of $2 \%$ potassium sulphate

Table 11. Effect of soil and foliar application of different N,P and K levels on biological yield $\left(\mathrm{Mg} / \mathrm{h}^{-1}\right)$

\begin{tabular}{|c|c|c|c|c|c|c|c|c|c|}
\hline \multirow{3}{*}{ Nitrogen } & \multirow{3}{*}{ Phosphorus } & \multicolumn{8}{|c|}{ Potassium } \\
\hline & & \multicolumn{4}{|c|}{ First season } & \multicolumn{4}{|c|}{ second season } \\
\hline & & $\mathbf{K}_{1}$ & $\mathbf{K}_{2}$ & $\mathbf{K}_{3}$ & Mean & $K_{1}$ & $\mathbf{K}_{2}$ & $\mathrm{~K}_{3}$ & Mean \\
\hline \multirow{3}{*}{$\mathbf{N}_{1}$} & $\mathbf{P}_{1}$ & 16.050 & 15.738 & 13.624 & 15.137 & 16.531 & 16.195 & 14.433 & 15.720 \\
\hline & $\mathbf{P}_{2}$ & 15.805 & 15.660 & 13.519 & 14.994 & 16.348 & 16.010 & 14.029 & 15.462 \\
\hline & $\mathbf{P}_{3}$ & 13.714 & 13.526 & 12.117 & 13.119 & 14.445 & 13.950 & 12.421 & 13.606 \\
\hline \multicolumn{2}{|l|}{ Mean } & 15.190 & 14.974 & 13.086 & 14.417 & 15.774 & 15.386 & 13.629 & 14.929 \\
\hline \multirow{3}{*}{$\mathbf{N}_{2}$} & $\mathbf{P}_{1}$ & 15.731 & 15.655 & 13.569 & 14.985 & 16.062 & 15.800 & 14.095 & 15.319 \\
\hline & $\mathbf{P}_{2}$ & 15.624 & 15.555 & 13.305 & 14.828 & 15.976 & 15.655 & 13.833 & 15.155 \\
\hline & $\mathbf{P}_{3}$ & 13.326 & 13.207 & 11.969 & 12.834 & 14.074 & 14.019 & 12.652 & 13.582 \\
\hline \multicolumn{2}{|l|}{ Mean } & 14.893 & 14.805 & 12.948 & 14.215 & 15.371 & 15.157 & 13.526 & 14.685 \\
\hline \multirow{3}{*}{$\mathbf{N}_{3}$} & $\mathbf{P}_{1}$ & 12.617 & 12.493 & 10.745 & 11.952 & 13.052 & 12.860 & 11.471 & 12.461 \\
\hline & $\mathbf{P}_{2}$ & 12.362 & 12.200 & 10.333 & 11.632 & 12.798 & 12.662 & 10.290 & 11.917 \\
\hline & $\mathbf{P}_{3}$ & 10.417 & 10.133 & 9.726 & 10.092 & 10.895 & 10.443 & 9.207 & 10.182 \\
\hline \multicolumn{2}{|l|}{ Mean } & 11.798 & 11.610 & 10.340 & 11.249 & 12.248 & 11.988 & 10.379 & 11.538 \\
\hline \multirow{3}{*}{ Mean of $P$} & $\mathbf{P}_{1}$ & 14.800 & 14.629 & 12.645 & 14.025 & 15.214 & 14.952 & 13.333 & 14.500 \\
\hline & $\mathbf{P}_{2}$ & 14.598 & 14.471 & 12.457 & 13.842 & 15.040 & 14.776 & 12.774 & 14.197 \\
\hline & $\mathbf{P}_{3}$ & 12.486 & 12.288 & 11.271 & 12.015 & 13.138 & 12.805 & 11.426 & 12.456 \\
\hline \multicolumn{2}{|l|}{ Mean of $\mathrm{K}$} & 13.962 & 13.795 & 12.124 & 13.294 & 14.464 & 14.179 & 12.512 & 13.718 \\
\hline \multicolumn{2}{|c|}{ L.S.D. at 0,05} & $\begin{array}{l}A=0.19 \\
A B=N S \\
B C=N S\end{array}$ & $\begin{array}{r}\mathbf{B}=0.24 \\
\mathrm{AC}= \\
\mathrm{ABC}=\end{array}$ & $\begin{array}{l}\text { C=0.2 } \\
\text { NS } \\
\text { NS }\end{array}$ & & $\begin{array}{l}\mathrm{A}=0.25 \\
\mathrm{AB}=\mathrm{NS} \\
\mathrm{BC}=\mathrm{NS}\end{array}$ & $\begin{array}{r}\mathbf{B}=\mathbf{0 . 3 0} \\
\mathrm{AC}= \\
\mathrm{ABC}=\end{array}$ & $\begin{array}{l}\mathrm{C}=0.24 \\
\mathrm{NS} \\
\mathrm{NS}\end{array}$ & \\
\hline
\end{tabular}

$\mathrm{N}_{1}=120 \mathrm{~kg} \mathrm{~N} /$ fed as soil application

$\mathrm{N}_{2}=90 \mathrm{~kg} \mathrm{~N} /$ fed as soil application + two time foliar spraying of $2 \%$ urea

$\mathrm{N}_{3}=60 \mathrm{~kg} \mathrm{~N} /$ fed as soil application + two time foliar spraying of $2 \%$ urea 
$\mathrm{P}_{1}=13.6 \mathrm{~kg} \mathrm{P} / \mathrm{fed}$ as soil application

$\mathrm{P}_{2}=6.8 \mathrm{~kg} \mathrm{P} / \mathrm{fed}$ as soil application + two time foliar spraying of $0.33 \% \mathrm{P}$

$\mathrm{P}_{3}=$ tow time foliar spraying of $0.33 \% \mathrm{P}$

$\mathrm{K}_{1}=39.9 \mathrm{~kg} \mathrm{~K} / \mathrm{fed}$ as soil application

$\mathrm{K}_{2}=19.9 \mathrm{~kg} \mathrm{~K} / \mathrm{fed}$ as soil application+ two time foliar spraying of $2 \%$ potassium sulphate

$\mathrm{K}_{3}=$ two time foliar spraying of $2 \%$ potassium sulphate

Table 12. Effect of soil and foliar application of different N,P and K levels on net income $\left(\mathrm{L} . \mathrm{E} / \mathrm{h}^{-1}\right)$

\begin{tabular}{|c|c|c|c|c|c|c|c|c|c|}
\hline \multirow{3}{*}{ Nitrogen } & \multirow{3}{*}{ Phosphorus } & \multicolumn{8}{|c|}{ Potassium } \\
\hline & & \multicolumn{4}{|c|}{ First season } & \multicolumn{4}{|c|}{ second season } \\
\hline & & $\mathbf{K}_{1}$ & $\mathbf{K}_{\mathbf{2}}$ & $\mathbf{K}_{3}$ & Mean & $\mathbf{K}_{1}$ & $\mathbf{K}_{2}$ & $\mathbf{K}_{\mathbf{3}}$ & Mean \\
\hline \multirow{3}{*}{$\mathbf{N}_{1}$} & $\mathbf{P}_{1}$ & 16952 & 17262 & 13765 & 15993 & 17325 & 17493 & 14907 & 16575 \\
\hline & $\mathbf{P}_{2}$ & 17233 & 17615 & 13894 & 16247 & 17431 & 17520 & 14630 & 16527 \\
\hline & $\mathbf{P}_{3}$ & 12000 & 12469 & 11085 & 11851 & 12908 & 13240 & 12215 & 12787 \\
\hline Mean & & 15395 & 15782 & 12915 & 14697 & 15888 & 16084 & 13917 & 15296 \\
\hline \multirow{3}{*}{$\mathbf{N}_{2}$} & $\mathbf{P}_{1}$ & 17400 & 17848 & 14359 & 16536 & 17245 & 17567 & 15153 & 16655 \\
\hline & $\mathbf{P}_{2}$ & 17736 & 18062 & 14443 & 16747 & 17580 & 17719 & 14882 & 16727 \\
\hline & $\mathbf{P}_{3}$ & 12529 & 16545 & 11833 & 13636 & 13628 & 13995 & 12310 & 13311 \\
\hline Mean & & 15888 & 17485 & 13545 & 15639 & 16151 & 16427 & 14115 & 15564 \\
\hline \multirow{3}{*}{$\mathbf{N}_{3}$} & $\mathbf{P}_{1}$ & 12190 & 12563 & 9309 & 11354 & 12781 & 13090 & 11485 & 12452 \\
\hline & $\mathbf{P}_{2}$ & 12366 & 12442 & 9586 & 11465 & 12961 & 13299 & 10815 & 12358 \\
\hline & $\mathbf{P}_{3}$ & 8140 & 8539 & 9445 & 8708 & 9437 & 9754 & 9388 & 9526 \\
\hline Mean & & 10899 & 11180 & 9447 & 10509 & 11726 & 12048 & 10563 & 11446 \\
\hline \multirow{3}{*}{ Mean of $P$} & $\mathbf{P}_{1}$ & 15514 & 15891 & 12478 & 14627 & 15784 & 16050 & 13848 & 15227 \\
\hline & $\mathbf{P}_{2}$ & 15779 & 16039 & 12641 & 14820 & 15990 & 16179 & 13442 & 15204 \\
\hline & $\mathbf{P}_{3}$ & 10890 & 12517 & 10788 & 11398 & 11991 & 12330 & 11304 & 11875 \\
\hline \multicolumn{2}{|l|}{ Mean of $\mathrm{K}$} & 14061 & 14816 & 11969 & 13615 & 14588 & 14853 & 12865 & 14102 \\
\hline \multicolumn{2}{|c|}{ L.S.D. at 0,05} & $\begin{array}{l}A=65.02 \\
A B=N S \\
B C=N S\end{array}$ & $\begin{array}{r}\mathbf{B}=1 \mathbf{1} \\
\mathbf{A C} \\
\mathrm{AB}\end{array}$ & $\begin{array}{l}25 \mathrm{C}= \\
\mathrm{NS} \\
=\mathrm{NS}\end{array}$ & 9.75 & $\begin{array}{l}A=75.19 \\
A B=N S \\
B C=N S\end{array}$ & $\begin{array}{r}B=6 \\
A C \\
A B\end{array}$ & \multicolumn{2}{|c|}{$\begin{array}{l}\text { C=55.96 } \\
\text { S }\end{array}$} \\
\hline
\end{tabular}

$\mathrm{N}_{1}=120 \mathrm{~kg} \mathrm{~N} /$ fed as soil application

$\mathrm{N}_{2}=90 \mathrm{~kg} \mathrm{~N} /$ fed as soil application + two time foliar spraying of $2 \%$ urea

$\mathrm{N}_{3}=60 \mathrm{~kg} \mathrm{~N} /$ fed as soil application + two time foliar spraying of $2 \%$ urea

$\mathrm{P}_{1}=13.6 \mathrm{~kg} \mathrm{P} / \mathrm{fed}$ as soil application

$\mathrm{P}_{2}=6.8 \mathrm{~kg} \mathrm{P} / \mathrm{fed}$ as soil application + two time foliar spraying of $0.33 \% \mathrm{P}$

$\mathrm{P}_{3}=$ tow time foliar spraying of $0.33 \% \mathrm{P}$

$\mathrm{K}_{1}=39.9 \mathrm{~kg} \mathrm{~K} / \mathrm{fed}$ as soil application

$\mathrm{K}_{2}=19.9 \mathrm{~kg} \mathrm{~K} / \mathrm{fed}$ as soil application+ two time foliar spraying of $2 \%$ potassium sulphate

$\mathrm{K}_{3}=$ two time foliar spraying of $2 \%$ potassium sulphate

\section{Nutrient uptake}

The effect of methods and levels of NPK fertilization on total $\mathrm{N}, \mathrm{P}$ and $\mathrm{K}$ uptake (in both grain and stover) were listed in Tables 13,14 and 15. The data reveal that total nitrogen uptake were significantly affected by nitrogen, phosphorus and potassium treatments, where $\mathrm{N}_{2}, \mathrm{P}_{1}$ or $\mathrm{P}_{2}$ as well as $\mathrm{K}_{1}$ or $\mathrm{K}_{2}$ treatments exhibited the highest nitrogen uptake in both season. On the other hand $\mathrm{N}_{3}, \mathrm{P}_{3}$ or $\mathrm{K}_{3}$ treatments recorded the lowest nitrogen uptake. The combinations between treatments show that the highest total nitrogen uptake was durned under $\mathrm{N}_{2}+$ $\mathrm{P}_{1}$ or $\mathrm{P}_{2}+\mathrm{K}_{1}$ or $\mathrm{K}_{2}$ treatment. On the other hand, $\mathrm{N}_{3}+$ $\mathrm{P}_{3}+\mathrm{K}_{3}$ treatment recorded the lowest total nitrogen uptake in both seasons.

As for total phosphorus uptake, the results indicate that $\mathrm{N}_{1}$ or $\mathrm{N}_{2}, \mathrm{P}_{2}$ as well as $\mathrm{K}_{1}$ or $\mathrm{K}_{2}$ treatments gave the highest phosphorus uptake, whereas, $\mathrm{N}_{3}, \mathrm{P}_{3}$ or $\mathrm{k}_{3}$ treatments exhibited the lowest total phosphorus uptake in both season. The interaction between treatments reveal that $\mathrm{N}_{1}$ or $\mathrm{N}_{2}+\mathrm{P}_{2}+\mathrm{K}_{1}$ or $\mathrm{K}_{2}$ treatment resulted in the highest total phosphorus uptake, whereas, the lowest total phosphorus uptake was recoded under the treatment of $\mathrm{N}_{3}+\mathrm{P}_{3}+\mathrm{K}_{3}$ in both seasons.

Concerning potassium uptake, the data show that total potassium uptake was significantly responded to $\mathrm{N}, \mathrm{P}$ and $\mathrm{K}$ treatments, where the highest total potassium uptake were recorded under $\mathrm{N}_{1}$ or $\mathrm{N}_{2}, \mathrm{P}_{1}$ or $\mathrm{P}_{2}, \mathrm{~K}_{1}$ or $\mathrm{K}_{2}$ treatments, while $\mathrm{N}_{3}, \mathrm{P}_{3}$ or $\mathrm{K}_{3}$ yielded the lowest total potassium uptake in both seasons. The interaction between the treatments revael that the highest potassium uptake was obtained due to N1 or $\mathrm{N}_{2}+\mathrm{P}_{1}$ or $\mathrm{P}_{2}+\mathrm{K}_{1}$ or $\mathrm{K}_{2}$ treatment, while the treatment of $\mathrm{N}_{3}+\mathrm{P}_{3}+\mathrm{K}_{3}$ treatment gave the lowest potassium uptake . The superiority of $\mathrm{N}_{2}, \mathrm{P}_{1}$ or $\mathrm{P}_{2}$ and $\mathrm{K}_{1}$ or $\mathrm{K}_{2}$ treatment on total nitrogen, phosphorus and potassium uptake over the other treatments is mainly due to its effect on both grains and stover yields as discussed before (Tables 7 and 8). In this concern, Halvin et al (2005) mentioned that foliar fertilizer is a form of liquid fertilizer through the foliage plants. This 
method tends to provide for rapid nutrient utilization. However, the response to foliar fertilizer only is often temporary due to minute amount applied nutrient, therefore combined foliar spraying with soil application gave the highest nutrient uptake.

$\underline{\text { Table 13. Effect of soil and foliar application of different N,P and K levels on total nitrogen uptake }\left(\mathrm{kg} / \mathrm{h}^{-1}\right)}$

\begin{tabular}{|c|c|c|c|c|c|c|c|c|c|}
\hline \multirow{3}{*}{ Nitrogen } & \multirow{3}{*}{ Phosphorus } & \multicolumn{8}{|c|}{ Potassium } \\
\hline & & \multicolumn{4}{|c|}{ First season } & \multicolumn{4}{|c|}{ second season } \\
\hline & & $\mathbf{K}_{1}$ & $\mathbf{K}_{2}$ & $\mathbf{K}_{3}$ & Mean & $\mathbf{K}_{1}$ & $\mathbf{K}_{2}$ & $\mathbf{K}_{3}$ & Mean \\
\hline \multirow{3}{*}{$\mathbf{N}_{1}$} & $\mathbf{P}_{1}$ & 196.90 & 191.81 & 166.31 & 185.01 & 202.76 & 200.45 & 177.12 & 193.44 \\
\hline & $\mathbf{P}_{2}$ & 193.38 & 193.48 & 164.86 & 183.90 & 200.62 & 196.60 & 171.95 & 189.72 \\
\hline & $\mathbf{P}_{3}$ & 166.76 & 162.86 & 161.64 & 163.75 & 176.00 & 168.36 & 150.43 & 164.93 \\
\hline \multicolumn{2}{|l|}{ Mean } & 185.69 & 182.71 & 164.26 & 177.56 & 193.12 & 188.48 & 166.50 & 182.70 \\
\hline \multirow{3}{*}{$\mathbf{N}_{2}$} & $\mathbf{P}_{1}$ & 229.83 & 229.69 & 198.83 & 219.45 & 238.24 & 234.48 & 208.88 & 227.20 \\
\hline & $\mathbf{P}_{2}$ & 230.17 & 229.07 & 193.81 & 217.68 & 237.02 & 230.88 & 203.45 & 223.79 \\
\hline & $\mathbf{P}_{3}$ & 194.43 & 192.24 & 173.79 & 186.82 & 206.38 & 206.19 & 184.64 & 199.07 \\
\hline \multicolumn{2}{|l|}{ Mean } & 218.14 & 217.00 & 188.81 & 207.98 & 227.21 & 223.86 & 199.00 & 216.69 \\
\hline \multirow{3}{*}{$\mathbf{N}_{3}$} & $\mathbf{P}_{1}$ & 165.45 & 163.74 & 138.86 & 156.02 & 170.10 & 168.26 & 148.86 & 162.40 \\
\hline & $\mathbf{P}_{2}$ & 162.29 & 159.76 & 136.79 & 152.94 & 166.76 & 165.29 & 136.55 & 156.20 \\
\hline & $\mathbf{P}_{3}$ & 135.60 & 131.14 & 126.81 & 131.18 & 141.19 & 135.40 & 119.50 & 132.03 \\
\hline \multicolumn{2}{|l|}{ Mean } & 154.45 & 151.55 & 134.14 & 146.71 & 159.36 & 156.31 & 134.98 & 150.21 \\
\hline \multirow{3}{*}{ Mean of $P$} & $\mathbf{P}_{1}$ & 197.40 & 195.07 & 168.00 & 186.83 & 203.69 & 201.07 & 178.29 & 194.35 \\
\hline & $\mathbf{P}_{2}$ & 195.29 & 194.10 & 165.14 & 184.84 & 201.48 & 197.60 & 170.64 & 189.90 \\
\hline & $\mathbf{P}_{3}$ & 165.60 & 162.07 & 154.07 & 160.58 & 174.52 & 169.98 & 151.52 & 165.34 \\
\hline \multicolumn{2}{|l|}{ Mean of $\mathrm{K}$} & 186.10 & 183.74 & 162.40 & 177.41 & 193.24 & 189.55 & 166.81 & 183.20 \\
\hline \multicolumn{2}{|c|}{ L.S.D. at 0,05} & $\begin{array}{l}\mathrm{A}=\mathbf{5 . 4 7} \\
\mathrm{AB}=\mathrm{NS} \\
\mathrm{BC}=\mathrm{NS}\end{array}$ & \multicolumn{3}{|c|}{$\begin{array}{l}\mathrm{B}=4.63 \\
\mathrm{AC}=\mathrm{NS} \\
\mathrm{ABC}=\mathrm{NS}\end{array}$} & $\begin{array}{l}A=5.68 \\
A B=N S \\
B C=N S\end{array}$ & \multicolumn{2}{|c|}{$\begin{array}{l}B=4.72 \\
A C=N S \\
A B C=N S\end{array}$} & \\
\hline
\end{tabular}

$\mathrm{N}_{1}=120 \mathrm{~kg} \mathrm{~N} /$ fed as soil application

$\mathrm{N}_{2}=90 \mathrm{~kg} \mathrm{~N} /$ fed as soil application + two time foliar spraying of $2 \%$ urea

$\mathrm{N}_{3}=60 \mathrm{~kg} \mathrm{~N} /$ fed as soil application + two time foliar spraying of $2 \%$ urea

$\mathrm{P}_{1}=13.6 \mathrm{~kg} \mathrm{P} /$ fed as soil application

$\mathrm{P}_{2}=6.8 \mathrm{~kg} \mathrm{P} / \mathrm{fed}$ as soil application + two time foliar spraying of $0.33 \% \mathrm{P}$

$\mathrm{P}_{3}=$ tow time foliar spraying of $0.33 \% \mathrm{P}$

$\mathrm{K}_{1}=39.9 \mathrm{~kg} \mathrm{~K} /$ fed as soil application

$\mathrm{K}_{2}=19.9 \mathrm{~kg} \mathrm{~K} / \mathrm{fed}$ as soil application+ two time foliar spraying of $2 \%$ potassium sulphate

$\mathrm{K}_{3}=$ two time foliar spraying of $2 \%$ potassium sulphate

$\underline{\text { Table 14. Effect of soil and foliar application of different N,P and } \mathrm{K} \text { levels on total phosphorus uptake }\left(\mathrm{kg} / \mathrm{h}^{-1}\right)}$

\begin{tabular}{|c|c|c|c|c|c|c|c|c|c|}
\hline \multirow{3}{*}{ Nitrogen } & \multirow{3}{*}{ Phosphorus } & \multicolumn{8}{|c|}{ Potassium } \\
\hline & & \multicolumn{4}{|c|}{ First season } & \multicolumn{4}{|c|}{ second season } \\
\hline & & $\mathbf{K}_{1}$ & $\mathbf{K}_{2}$ & $\mathbf{K}_{3}$ & Mean & $\mathbf{K}_{1}$ & $\mathbf{K}_{2}$ & $\mathbf{K}_{3}$ & Mean \\
\hline \multirow{3}{*}{$\mathbf{N}_{\mathbf{1}}$} & $\mathbf{P}_{1}$ & 55.29 & $\begin{array}{l}53.74 \\
\end{array}$ & 45.29 & 51.44 & $\mathbf{5 6 . 5 5}$ & 55.31 & 49.50 & 53.79 \\
\hline & $\mathbf{P}_{2}$ & 68.33 & 69.26 & 60.81 & 66.13 & 72.55 & 72.02 & 61.79 & 68.79 \\
\hline & $\mathbf{P}_{3}$ & 53.86 & 53.02 & 47.14 & 51.34 & $\begin{array}{ll}57.88 \\
\end{array}$ & 56.21 & 48.05 & 54.05 \\
\hline Mean & & 59.17 & 58.67 & 51.07 & 56.30 & 62.33 & 61.21 & 53.12 & 58.89 \\
\hline \multirow{3}{*}{$\mathbf{N}_{2}$} & $\mathbf{P}_{1}$ & 55.86 & 55.83 & 47.31 & 53.00 & 55.86 & 55.14 & 49.86 & 53.62 \\
\hline & $\mathbf{P}_{2}$ & 69.24 & 68.12 & $\mathbf{5 8 . 5 0}$ & 65.29 & 70.98 & 71.24 & 62.07 & 68.10 \\
\hline & $\mathbf{P}_{3}$ & 53.12 & 51.79 & 47.98 & 50.96 & $\mathbf{5 4 . 5 5}$ & 55.76 & 50.50 & 53.60 \\
\hline Mean & & 59.40 & 58.67 & 51.26 & 56.44 & 60.45 & 60.71 & 54.14 & 58.44 \\
\hline \multirow{3}{*}{$\mathbf{N}_{3}$} & $\mathbf{P}_{1}$ & 42.55 & 41.50 & $\mathbf{3 4 . 5 5}$ & 39.53 & 42.95 & 43.71 & 38.50 & 41.72 \\
\hline & $\mathbf{P}_{2}$ & 53.90 & 53.62 & 44.64 & 50.72 & 56.17 & 56.38 & 45.76 & 52.77 \\
\hline & $\mathbf{P}_{3}$ & 40.31 & 39.10 & 37.81 & 39.07 & 42.71 & 40.19 & 37.02 & 39.98 \\
\hline Mean & & 45.60 & 44.74 & 39.00 & 43.11 & 47.29 & 46.76 & 40.43 & 44.83 \\
\hline \multirow{3}{*}{ Mean of $P$} & $\mathbf{P}_{1}$ & 51.24 & 50.36 & 42.38 & 47.99 & 51.79 & 51.43 & 45.95 & 49.72 \\
\hline & $\mathbf{P}_{2}$ & 70.98 & 63.67 & 64.31 & 66.32 & 66.57 & 66.55 & 56.55 & 63.22 \\
\hline & $\mathbf{P}_{3}$ & 49.10 & 47.98 & 44.31 & 47.13 & 51.71 & 50.71 & 45.19 & 49.21 \\
\hline \multicolumn{2}{|l|}{ Mean of K } & 57.10 & 54.00 & 49.98 & 53.69 & 56.69 & 56.24 & 49.24 & 54.06 \\
\hline \multicolumn{2}{|c|}{ L.S.D. at 0,05} & $\begin{array}{l}A=3.34 \\
A B=N S \\
B C=N\end{array}$ & \multicolumn{3}{|c|}{$\begin{array}{c}B=3.79 \\
A C=N S \\
A B C=N S\end{array}$} & $\begin{array}{l}\mathrm{A}=3.96 \\
\mathrm{AB}=\mathrm{NS} \\
\mathrm{BC}=\mathrm{NS}\end{array}$ & \multicolumn{3}{|c|}{$\begin{array}{c}\mathrm{B}=3.24 \\
\mathrm{AC}=\mathrm{NS} \\
\mathrm{ABC}=\mathrm{NS}\end{array}$} \\
\hline
\end{tabular}


$\mathrm{N}_{1}=120 \mathrm{~kg} \mathrm{~N} /$ fed as soil application

$\mathrm{N}_{2}=90 \mathrm{~kg} \mathrm{~N} /$ fed as soil application + two time foliar spraying of $2 \%$ urea

$\mathrm{N}_{3}=60 \mathrm{~kg} \mathrm{~N} /$ fed as soil application + two time foliar spraying of $2 \%$ urea

$\mathrm{P}_{1}=13.6 \mathrm{~kg} \mathrm{P} / \mathrm{fed}$ as soil application

$\mathrm{P}_{2}=6.8 \mathrm{~kg} \mathrm{P} / \mathrm{fed}$ as soil application + two time foliar spraying of $0.33 \% \mathrm{P}$

$\mathrm{P}_{3}=$ tow time foliar spraying of $0.33 \% \mathrm{P}$

$\mathrm{K}_{1}=39.9 \mathrm{~kg} \mathrm{~K} / \mathrm{fed}$ as soil application

$\mathrm{K}_{2}=19.9 \mathrm{~kg} \mathrm{~K} /$ fed as soil application+ two time foliar spraying of $2 \%$ potassium sulphate

$\mathrm{K}_{3}=$ two time foliar spraying of $2 \%$ potassium sulphate

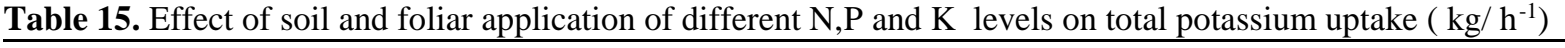

\begin{tabular}{|c|c|c|c|c|c|c|c|c|c|}
\hline \multirow{3}{*}{ Nitrogen } & \multirow{3}{*}{ Phosphorus } & \multicolumn{8}{|c|}{ Potassium } \\
\hline & & \multicolumn{4}{|c|}{ First season } & \multicolumn{4}{|c|}{ second season } \\
\hline & & $\mathbf{K}_{1}$ & $\mathbf{K}_{2}$ & $\mathbf{K}_{\mathbf{3}}$ & Mean & $\mathbf{K}_{1}$ & $\mathbf{K}_{2}$ & $\mathbf{K}_{3}$ & Mean \\
\hline \multirow{3}{*}{$\mathbf{N}_{1}$} & $\mathbf{P}_{1}$ & 196.00 & 214.64 & 183.50 & 198.05 & 211.45 & 230.05 & 193.76 & 211.75 \\
\hline & $\mathbf{P}_{2}$ & 193.33 & 214.02 & 180.83 & 196.06 & 207.26 & 226.64 & 189.71 & 207.87 \\
\hline & $\mathbf{P}_{3}$ & 171.60 & 185.60 & 161.98 & 173.06 & 187.12 & 199.14 & 168.48 & 184.91 \\
\hline Mean & & 186.98 & 204.74 & 175.43 & 189.05 & 201.95 & 218.62 & 183.98 & 201.52 \\
\hline \multirow{3}{*}{$\mathbf{N}_{2}$} & $\mathbf{P}_{1}$ & 190.19 & 211.79 & 180.02 & 194.00 & 202.40 & 222.98 & 188.45 & 204.61 \\
\hline & $\mathbf{P}_{2}$ & 188.52 & 211.12 & 176.83 & 192.16 & 201.67 & 221.60 & 185.19 & 202.82 \\
\hline & $\mathbf{P}_{3}$ & 164.24 & $\mathbf{1 8 0 . 4 5}$ & 159.52 & 168.07 & 179.86 & 199.38 & 170.90 & 183.38 \\
\hline Mean & & 180.98 & 201.12 & 172.12 & 184.74 & 194.64 & 214.64 & 181.52 & 196.94 \\
\hline \multirow{3}{*}{$\mathbf{N}_{3}$} & $\mathbf{P}_{1}$ & 154.86 & 171.90 & 144.74 & 157.17 & 167.52 & 183.05 & 154.74 & 168.44 \\
\hline & $\mathbf{P}_{2}$ & 153.10 & 166.88 & $\mathbf{1 4 1 . 4 5}$ & 153.81 & 163.81 & $\mathbf{1 8 0 . 4 5}$ & 141.21 & $\mathbf{1 6 1 . 8 3}$ \\
\hline & $\mathbf{P}_{3}$ & 131.31 & 140.14 & 129.29 & 133.58 & 141.93 & 149.60 & 125.60 & 139.04 \\
\hline Mean & & 150.93 & 159.64 & 138.50 & 149.69 & 157.76 & 171.02 & 140.52 & 156.44 \\
\hline \multirow{3}{*}{ Mean of $P$} & $\mathbf{P}_{1}$ & 180.36 & 199.45 & $\mathbf{1 6 9 . 4 3}$ & 183.08 & 193.79 & 212.02 & 178.98 & 194.93 \\
\hline & $\mathbf{P}_{2}$ & 178.31 & 197.33 & 166.38 & 180.67 & 190.90 & 209.57 & 172.05 & 190.84 \\
\hline & $\mathbf{P}_{3}$ & 155.71 & 168.74 & 150.26 & 158.24 & 169.64 & 182.71 & 155.00 & 169.12 \\
\hline Mean of $\mathrm{K}$ & & 171.45 & 188.50 & 162.02 & 173.99 & 184.79 & 201.43 & 168.67 & 184.96 \\
\hline \multicolumn{2}{|c|}{ L.S.D. at 0,05} & $\begin{array}{l}A=2.25 \\
\mathrm{AB}=\mathrm{NS} \\
\mathrm{BC}=\mathrm{NS}\end{array}$ & \multicolumn{3}{|c|}{$\begin{array}{c}B=2.03 \quad C= \\
A C=N S \\
A B C=N S\end{array}$} & $\begin{array}{l}\mathrm{A}=2.96 \\
\mathrm{AB}=\mathrm{NS} \\
\mathrm{BC}=\mathrm{NS}\end{array}$ & \multicolumn{3}{|c|}{$\begin{array}{c}B=2.16 \quad C \\
A C=N S \\
A B C=N S\end{array}$} \\
\hline
\end{tabular}

$\mathrm{N}_{1}=120 \mathrm{~kg} \mathrm{~N} /$ fed as soil application

$\mathrm{N}_{2}=90 \mathrm{~kg} \mathrm{~N} /$ fed as soil application + two time foliar spraying of $2 \%$ urea

$\mathrm{N}_{3}=60 \mathrm{~kg} \mathrm{~N} /$ fed as soil application + two time foliar spraying of $2 \%$ urea

$\mathrm{P}_{1}=13.6 \mathrm{~kg} \mathrm{P} / \mathrm{fed}$ as soil application

$\mathrm{P}_{2}=6.8 \mathrm{~kg} \mathrm{P} / \mathrm{fed}$ as soil application + two time foliar spraying of $0.33 \% \mathrm{P}$

$\mathrm{P}_{3}=$ tow time foliar spraying of $0.33 \% \mathrm{P}$

$\mathrm{K}_{1}=39.9 \mathrm{~kg} \mathrm{~K} / \mathrm{fed}$ as soil application

$\mathrm{K}_{2}=19.9 \mathrm{~kg} \mathrm{~K} / \mathrm{fed}$ as soil application+ two time foliar spraying of $2 \%$ potassium sulphate

$\mathrm{K}_{3}=$ two time foliar spraying of $2 \%$ potassium sulphate

\section{Conclusion}

It could be concluded that mixed of $\mathrm{N}_{2}+\mathrm{P}_{2}+\mathrm{K}_{2} 90$ $\mathrm{kg} \mathrm{N} /$ fed as soil application with foliar spraying of $2 \%$ urea solution two time mixed $6.8 \mathrm{~kg} \mathrm{P} / \mathrm{fed}$ as soil application with foliar spraying of $0.33 \%$ two time + combined $19.9 \mathrm{~kg} \mathrm{~K} /$ fed as soil application with foliar spraying of $2 \%$ potassium sulphate solution two time gave the highest net income of maize plant under the studied soil.

\section{References}

Abd El-Hafeez, A.M.; H.A. Awadalla and S.A. Ismail 2013: Influence of different sources and levels of nitrogen and rock phosphate addition on maize productivity and soil fertility. J. Soil Sci. and Agric Eng., Mansoura Univ., 4(11):1313-1328.
Allam, S. M. M.; A. S. Osman.; M. Y. Gbraiel and G. M. El- Shebing, 2001:

Effect of nitrogen rates and foliar application of zinc and boron on maize. Egypt. J. Appl. Sei.,16:161168.

Ali, M. E.; S. A. Ismail; A. H. Abd El-Hameid; O.H.M. El-Hussieny and F.H. El-Sherf, 2012: Effect of natural fertilizers under different levels of nitrogen and farmyard manure on the productivity of maize. Fayoum J.Agric. Res. \& Dev.; 26(1):49 $-63$.

Amhakian, S. O. and I. O. Osemwota, 2012: Effects of different levels of phosphorus on the performance of Maize (Zea mays L) in Anyigba, North central State, Nigeria. Soil. Ambrose Alli Univ. Expome., 15(2):1049- 1058.

Anonymous, A. M. 1985: International rules for Seed. Testing. Seed Sci. Tech., 13: 299- 320. 
Crozier, C R.; J. L. Havlin; G. D. Hoyt; J. W. Rideout and R. Mcdaniel, 2009: Three experimental systems to evaluate phosphorus supply from enhanced granulated manure ash. Agro. J. 101(4): 880- 888.

Chapman, H. D. and F. Pratt, 1961: Methods of Analysis of Soils, Plants and Water. Uni. Calf.

Dahiru, T. M.; N. B. Kwaga and M. Yamma, 2016: Effect of foliar fertilizer (boost-extra) and NPK levels on vegetative growth of maize (Zea mays L.) grown in Mubi, Northern Guinea savannah zone of Nigeria. Archives of Applied Science Research, 8(7): 16-21.

El-Azab, M. E., 2015: Increasing $\mathrm{Zn}$ ratio in a compound foliar NPK fertilizer in relation to growth, yield and quality of corn plant. Journal of Innovations in Pharmaceuticals and Biological Sci., 2(4); 451-468.

Femandez E. C. M. ; P. A. Sanchez, 1990: The role of organic inputs and soil organic matter for nutrient cycling in tropical soil In : Asadu. C. L. A. Egbobe O. B. and Asiedu R. (eds). Storage Life of Eleven Cultivars of white Yam (Dioscorea Rotundala) grown with or without NPK Fertilizer and the Relationship with Residual Soil NPK. J. Trop. Agric. Food Environ Ext. 7(3) : 235 - 241.

Gebraiel, M. Y.; M. N. Gohar; F. S. Salem and H. W. A. Wahba, 2005: Vegetative growth and yield of maize (Zea mays L.). as affected by nitrogen, potassium and zinc fertilization. Egypt. J. Appl. Sci., 20(2B): 739-755.

Gul, S.; M. H. Khan; B. A. Khanday, and S. Nabi, 2015: Effect of sowing methods and NPK levels on growth and yield of rainfed maize (Zea mays L.), Agro. Sher-e-Kashmir Univ. Agri. Scie.

Havlin J. L.; D. B, James ; L. T. Samuel and L. N. Werner 2005: Soil Fertility and Fertilizers:An Introduction to Nutrient Management (7th Edition). New Delhi 10020391.

Hussain, N.; A. Z. Kan; H. Akhar; N. G. Bangash; Z. H. Kan and M. Drees, 2007: Response of Maize varieties to phosphorus and potassium levels. Sarhnd Agirc., 23(4): 881-888.

Hu, Y.; B. Z, and S. Urs, 2008: Effect of foliar fertilization application on the growth and mineral nutrient content of maize seedlings under drought and salinity. Agric. Pannon Univ. Georgion Faculty Soil Science and Plant Nutrition. 24: 133141.

Ismail, S. A.; M.A. Morsy; A. A. Omran and M. M. Foaad, 2006: The productivity of some hybrids (Zea mays L.) grown in an alluvial soil under different nitrogen sources and levels. The Second Conference of Farm Integrated Pest Management, Fac. of Agric., Fayoum Univ., 16-18 January,

Khan, A. ; I. Khan,; Z. Shah and Z. Hussain, 2014: Growth and yield response of maize (Zea mays L.) to foliar NPK fertilizers under moisture stress condition. Agro. Univ. Agric. Peshawar , Soil Environ, 33(2): 116-123.

Kruczek, A., 2005: Phosphorus utilization from fertilizer and accumulation mineral components in the initial stage of maize development. Polish J. Envir. Stuies., 14(4): 467-475.

Kubar, S.; Z. Hassan; A. N. Shah; I. Rajpar and S. A. Qureshi, 2013: Response of maize to novel organic potassium fertilizer developed from fruit and vegetable wastes., Pak. J. Agni. Eng., Vet. Sci., 29(1): 1- 12.

Maqsood, M.; M. A. Shehzad; A.A. Wahid and A. A. Butt, 2013: Improving droght tolerance in maize (Zea mays) with potassium application in furrow irrigation system. Paki. J. Agric. Biol., 15(6) : 1193-1198.

Markus, D. K.; J. P. Mckinnon and A. F. Baccofuri, 1982: Atomated analysis of nitrate and ammonium in soil. New Jersey Agric. Exp. Stm. Publication No. D/15117. 84. Supported by Funs, Presented in Port Berore Dive 5.4 Soil Sci. of Mer., Anahein, (A,Des.2k 1982).

Mengel, K. and E Kirby 1987: Principles of Plant Nutrition. International Potash Institute, P. O. Box. Ch. 3084, warbling Fen - Bern, Switzerland

Piper, C. S. (1950). Soil and plant analysis. interscience pub. imc. new york.

Parasuraman, P.; R. Prakash and C. V. Chandrasekaran, 2008: Response of hybrid maize (Zea mays L.) to soil and foliar application of nutrients. Madras Agric. J., 95 (1/6) 200-202.

Sadik, M.K.; S.A. Ismail; O.H.M. El-Hussieny and R.F. Hashem, 2009: Influence of levels and methods of some organic and inorganic fertilization application on maize 1-Growth and nutrients uptake. J. Agric. Sci., Mansoura Univ., 34(7):9001-9014

Salwau, M. I. M. and G. M. Shams El-Din, 1992: Effect of nitrogen fertilization and defoliation on the yield and yield components of maize. $5^{\text {th }}$ Conf. of Agron., 13-15 Sept., Zagazig Univ.

Sarhan, M. G. R., 2006: Effect of nitrogen sources and levels on the accumulation and distribution of nitrate in plant, soil and groundwater. M. Sc. Thesis, Fac of Agric., Minia Univ., Egypt.

Siam, H. S.; M. G. Abd - El - Kader and H. I. El Alia, 2008: Yield and yield components of maize as affected by different sources and application rates of nitrogen fertilizer . ARPN J. Agri. Biological Sc., 5(1): 29-33.

Sidrak, N.N., 2007: Effect of water stress, planting dates and potassium fertilization on corn crop (Zea mays L.) Ph.D. Thesis, Fac. of Agric., Zagazig Univ.

Thirupathi , G. E. ; CH. V. Sugar and K. B. S. Devi, 2016: Effect of nitrogen and sulphur levels on growth, yield quality and economics of single cross hybrid maize (Zea mays L ) International Journal of Science, Environment and Technology , 5(5) : $2989-2998$. 
Yosefi, A.; M. Galawi; M. Ramrodi and R. Mousargis, 2011: Effect of phosphours and chmical phosphours fertilizer accompanied with macro nutrient foliar application on growth, yield and yield components of maize . Agro. Zabol Univ., 5(2) : 175- 180.
Zorkany, E. S. K., 2014: Scheduling irrigation of corn (Zea mays) using the evaporation pan method under different potassium. Ph. D. Thesis, Fac. of Agric., Minia Univ. 


\section{تداعيات التسميد الارضى والورقي لعناصر النيتروجين والفوسفور والبوتاسيوم على محصول وصافى الاخل لنبات الذرة الناميةعلى الاراضى الطينية الرسويية

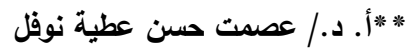

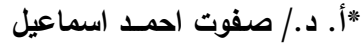 \\ * مركز البحوث الزراعية

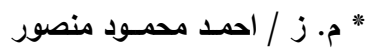

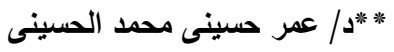

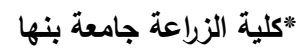

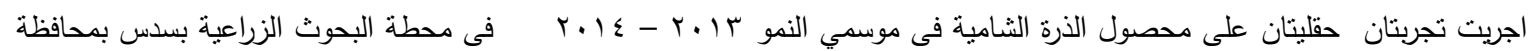

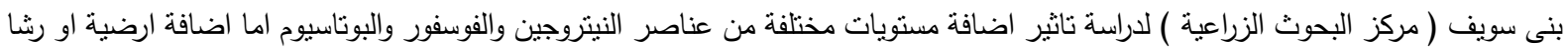

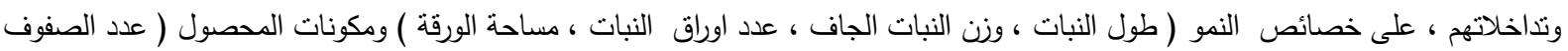

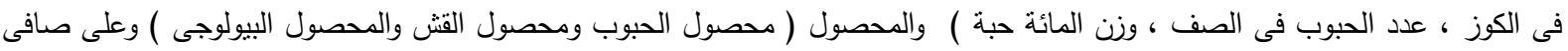
دخل محصول الحبوب والامتصاص الكلى ( فى الحبوب والقش ) لعناصر النيتروجين والفوسفور والبوناسيوم . وكانت معاملات التجربة كما يلى :معاملات النيتروجين :

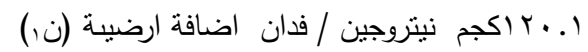

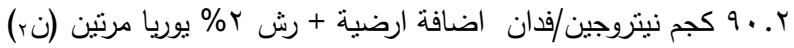

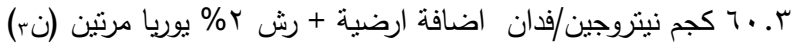

معاملات القوسفور :

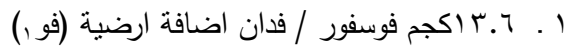

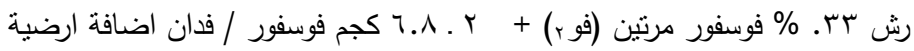

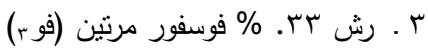

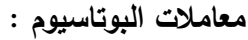

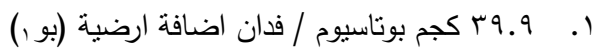

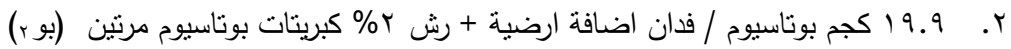

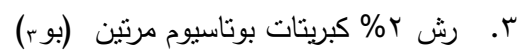

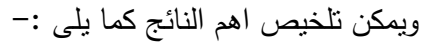

ادت المعاملة (ن () الى اعلى قيم لطول النبات ومساحة الورقة والوزن الجاف للنبات وعدد الصفوف في الكوز ووزن المائة حبة ومن ناحية اخرى

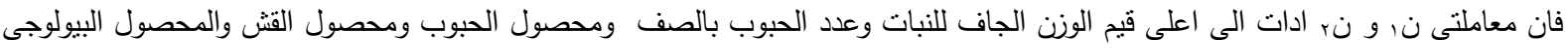
والامتصاص الكلى للفوسفور والبوتاسيوم ، بينما اعلى امتصاص للنيتروجين الكلى وصافى الدخل كان للمعاملة (ن) ، ولم يناثز عدد اوراق النبات

بمعاملات النيتروجين - ادت معاملات فو او فو ب او بو , او بو ب الى اعلى قيم طول النبات والوزن الجاف للنبات ومساحة الورقة وعدد الصفوف فى الكوز وعدد الحبوب

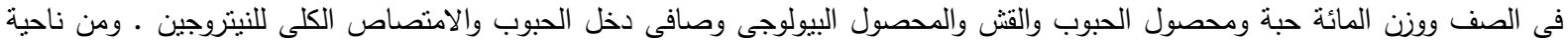
اخرى فقد ادت معاملات فو r ، بو r الى اعلى قيم الامتصاص للفوسفور والبوتاسيوم على الترتيب بينما لم يتاثر عدد اوراق النبات بمعاملات الفوسفور والبوتاسيوم • - ادت معاملات نץ ، فو بهبو ب الى اقل القيم من خصائص النمو والمحصول ومكوناتة وصافى الدخل وامتصاص العناصر . - عموما فان معاملة ن, او نr + فو , او فوب + بو , او بو ب ادت الى الحصول على اعلى قيم للصفات المدروسة .

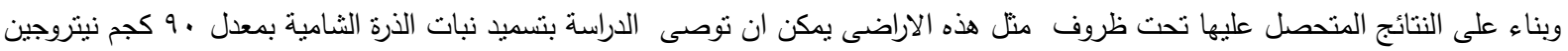

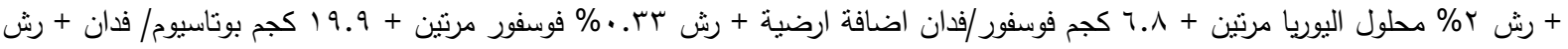

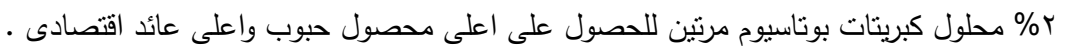

